\title{
On the Performance of Bidirectional NOMA-SWIPT Enabled IoT Relay Networks
}

\author{
Ashish Rauniyar, Member, IEEE, Paal Engelstad, Senior Member, IEEE, and Olav N. Østerbø, Senior \\ Member, IEEE
}

\begin{abstract}
In this paper, a bidirectional relaying (BR) nonorthogonal multiple access (NOMA) with simultaneous wireless information and power transfer (SWIPT) termed as BR NOMASWIPT is proposed and investigated for the Internet of Things (IoT) relay networks. Here, multiple NOMA users in one group can communicate or exchange information with multiple NOMA users in another group through a common energy harvesting (EH) based relay. The EH based relay exploits the radio frequency (RF) energy supplied by the two NOMA user groups to recharge itself, and then it exchanges the information between them. Specifically, the two groups of NOMA users transmit the information intended for the exchange to the relay node using the uplink NOMA protocol. The relay node first harvests the RF energy through the signals of the two group of NOMA users, and then it carries out the exchange of information between two NOMA user groups by using the downlink NOMA protocol. To the best of our knowledge, there is no existing study or research on BR NOMA with SWIPT. Therefore, in this paper, we examine in detail, the performance of BR NOMA-SWIPT enabled IoT relay networks. Specifically, we study a popular EH time switching (TS) relaying architecture with BR and NOMA. We also study the effect of both perfect successive interference cancellation (pSIC) and imperfect SIC (ipSIC) on the proposed BR NOMA-SWIPT system. Analytical expressions for the outage probability and ergodic capacity are mathematically derived. The analytical results of our proposed system model are validated by the simulation results, and representative performance comparisons are presented thoroughly, which not only provides practical insights into the effect of different system parameters on the overall network performance, but it also demonstrates that our proposed BR NOMA-SWIPT can attain significant throughput and capacity gains as compared to conventional BR multiple access schemes.
\end{abstract}

Index Terms-Radio frequency, NOMA, energy harvesting, SWIPT, bidirectional, relaying, time switching, ergodic capacity, outage probability.

\section{INTRODUCTION}

$\mathbf{T}$ HE proliferation of technologies like Internet of Things (IoT) and Industrial IoT (IIoT) has led to rapid growth in the number of connected devices and the volume of data associated with IoT applications [1]. Reference [2] has predicted that there will be 125 billion IoT devices connected to the Internet by 2030. The current underlying wireless network is based on orthogonal multiple access (OMA) by

A. Rauniyar and P. Engelstad are with the Autonomous Systems and Sensor Technologies Research Group, Department of Technology Systems, University of Oslo, Oslo, 0316, Norway and Autonomous System and Network (ASN) Research Group, Department of Computer Science, Oslo Metropolitan University, Oslo, 0130, Norway (e-mail: ashish@oslomet.no;paalen@oslomet.no).

O. N. Østerbø is with Telenor Research, Oslo, 1360, Norway (e-mail: olav.osterbo@getmail.no).

Accepted For Publication in IEEE Sensors Journal assigning orthogonal resources to multiple users. OMA cannot serve multiple IoT devices simultaneously and maximize the resource efficiency. Hence, OMA is considered spectrally inefficient for the design and optimization of the next-generation wireless systems [3][4]. To provide massive connectivity to billions of small IoT sensor and devices and to ameliorate their capacity demands, non-orthogonal multiple access (NOMA) has been considered as a potential candidate for the upcoming fifth-generation (5G) and next-generation networks [5][6][7]. Fundamentally, in NOMA, multiple signals or messages for users with distinct channel conditions are multiplexed in power domain at the same time, frequency and code in order to achieve a balanced trade-off between system throughput and user fairness. Specifically, in power-domain NOMA, when the user that have been assigned low power due to strong channel conditions, receives the NOMA signal, it first decodes the symbol of the users that have been assigned more power due to worse channel conditions. Then, it subtracts it to decode its own symbol. Therefore, through this process, users can be separated at the receiver side, which is popularly known as successive interference cancellation (SIC) [8][9].

Moreover, in addition to improving the spectral efficiency (SE), which is the main motivation of NOMA, another key objective of the next-generation wireless networks, is to maximize the energy efficiency so as to support massive IoT device communication and data transmission. To this end, simultaneous wireless information and power transfer (SWIPT) has been contemplated as an energy efficient viable solution to selfsustainable and green communication in IoT relay networks [10][11]. Since radio frequency (RF) signals carry both energy and information simultaneously, the IoT relay nodes can recharge themselves through energy harvesting $(\mathrm{EH})$ from the RF signal and at the same time decode the information data and then relay it to the destination nodes by utilizing the harvested energy [12]. However, due to practical constraints of EH circuits, it cannot be directly applied for information decoding (ID) at the same time [13]. Thus, according to the signal partition method for $\mathrm{EH}$ and ID, time switching relaying (TSR) and power splitting relaying (PSR) are two popular EH architectures widely considered for SWIPT [14]. In this paper, we focus on the TSR architecture because of its low complexity and ease of implementation [15]. However, it should be noted that our proposed system model can be easily extended to other EH architectures, such as the PSR [16] and the hybrid TSR-PSR architectures [17]. 


\section{RELATED WORKS AND CONTRIBUTIONS}

NOMA has been widely studied with cooperative relaying to combat wireless impairments, such as fading and other environmental factors, to improve the system capacity and reliability of wireless networks [18][19]. However, the cooperation for relaying comes at an extra energy consumption of the relay nodes, which may be battery operated in the context of IoT, and this may prevent it from taking an active part in cooperative relaying. Due to significant SE of NOMA, as compared to OMA, NOMA has also been extensively researched in combination with other technologies, such as cooperative NOMA [20] and NOMA-SWIPT [21][22].

A two-phase cooperative relaying strategy was proposed using the concept of uplink and downlink NOMA in [23] where the authors successfully analyzed the capacity and outage probability of a dual-hop decode-and-forward (DF) relayaided NOMA scheme. In downlink NOMA, the strong channel users achieve throughput gains by successively decoding and cancelling the messages of the weak channel users, prior to decoding their own signals. In the uplink NOMA, the BS successively decodes and cancels the messages of strong channel users before decoding the signals of weak channel users to enhance the throughput of weak channel users [24]. A comprehensive difference between uplink and downlink NOMA is given in [25]. A full/half duplex user relaying scheme in NOMA systems was proposed in [26] where strong NOMA users act as relays for weak NOMA users. Further, the authors in [27] proposed a novel receiver design for cooperative NOMA systems where dedicated relays are used to assist NOMA users. Among several research directions of NOMA in cooperative networks, NOMA-SWIPT is being considered as the most promising active research area by researchers for the development of upcoming next-generation wireless networks. A cooperative network where a source node communicates with two NOMA users through an EH based relay was analyzed in [28] to investigate the impact of power allocation policies in NOMA-SWIPT networks. Joint power allocation and time switching control for energy efficiency optimization in a TS-based NOMA-SWIPT system was proposed in [29]. A SWIPT-aided NOMA transmission scheme to support energyefficient uplink NOMA transmissions, helping the source node in receiving the signals with distinguished power levels, was proposed in [30]. However, all of these schemes are only based on a one way relaying (OWR) scheme where messages are relayed or transmitted only in one direction. Two-way relaying (TWR) or bidirectional relaying (BR) where two users can simultaneously exchange information through a common relay has gained much attention because of its high SE as compared to OWR [31].

\section{A. Motivations and Contributions}

Aiming to increase the SE further, NOMA and BR can be integrated together. There are already a few proposals that have successfully applied NOMA for BR, such as [32][33][34]. The algorithm to find the optimal power allocation that maximizes the user fairness and sum-rate was studied in [32] for a bidirectional cooperative NOMA in a two user scenario without full channel state information. Here, the bidirectional cooperation was considered between two NOMA users for improved decoding of the signal of the user that is not performing the SIC. A TWR-NOMA system was investigated in [33] where two groups of NOMA users can exchange the messages with the aid of one half-duplex decode-andforward (DF) relay. The authors also investigated the effect of imperfect SIC (ipSIC) and perfect SIC (pSIC) on a TWRNOMA system. With the model presented in [33], the authors in [34] proposed a similar BR-NOMA model where they studied the ergodic sum capacity and outage capacity to evaluate the performance of the system. All these models have only integrated BR in cooperative NOMA networks. Moreover, these works have ignored the impact of EH in their considered network. To the best of our knowledge, there is no existing work or contribution on BR for NOMA-SWIPT networks. The reason for combining BR and NOMA with SWIPT in cooperative networks is obvious and simple, as bidirectional NOMA improves the SE and SWIPT provides incentives to the IoT relay node through $\mathrm{RF} \mathrm{EH}$ to take an active part in relaying. In summary, the major contribution of our paper are as follows:

- We propose and examine in detail, the performance of BR NOMA-SWIPT enabled IoT relay networks where users in one NOMA group can exchange information with other users in another NOMA group with the help of an energy harvesting based relay node $R$.

- The outage probability, throughput, and ergodic capacity of each user and the sum-throughput and ergodic sum capacity of the proposed system are analytically derived under the both pSIC and ipSIC scenarios.

- For a fair comparison and benchmarking of our results, we devise and investigate the TWR/BR-OMA SWIPT system considering time division multiple access (TDMA) and compare it with our proposed system model. This demonstrates the significant improvement in the throughput and ergodic capacity of our BR NOMASWIPT system.

- We also study the effect of user pairing for the exchange of information in different groups and show the sum capacity enhancement of the BR NOMA-SWIPT system.

- We provide thorough practical insights into the effect of different system parameters on the overall network performance. We also show that our derived analytical results match exactly with the simulation results, to demonstrate that our analytical derivations are correct.

\section{B. Organization}

The rest of the paper is organized as follows. In Section III, we present our system and channel model for the BR NOMASWIPT enabled IoT relay network. In Section IV, we present our system model based on a time switching EH architecture with a detailed explanation of the first stage and second stage. The analysis of the outage probability, sum-throughput, ergodic capacity and ergodic sum capacity of the proposed system along with its analytical derivations are carried out in Section V. Numerical results and discussions are presented in 


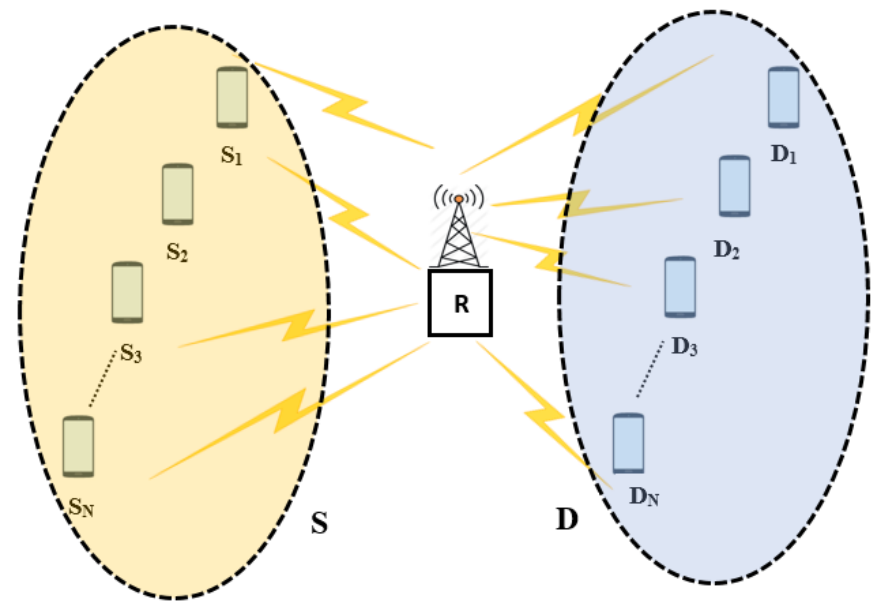

Fig. 1. Generic reference system model for BR NOMA with SWIPT

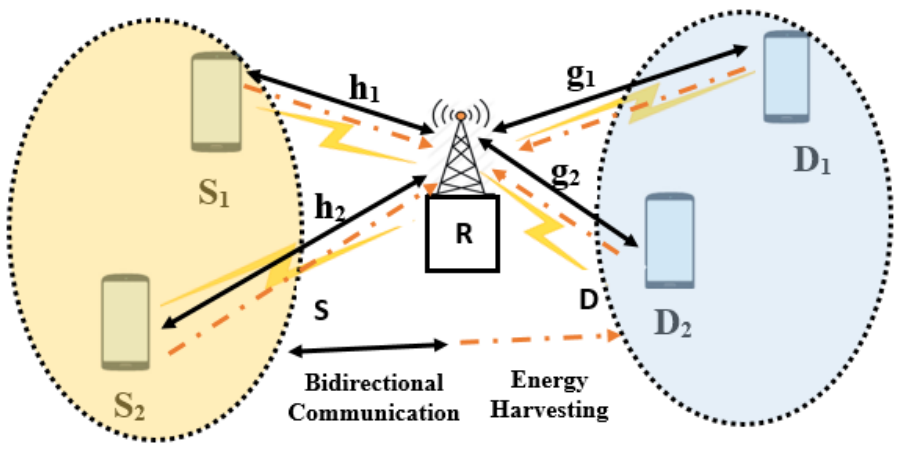

Fig. 2. Proposed system model scenario

Section VI. Conclusions and future works are discussed in Section VII.

\section{System ModeL}

A generic reference model for our considered scenario is shown in Fig. 1. It consists of multiple NOMA users in group $S=\left\{S_{1}, S_{2}, S_{3}, \ldots \ldots, S_{N}\right\}$ and multiple NOMA users in group $D=\left\{D_{1}, D_{2}, D_{3}, \ldots . ., D_{N}\right\}$, which can exchange information and communicate via the bidirectional IoT relay node R. Here the groups $S$ and $D$ form the two NOMA groups with $N$ number of NOMA users in each group. It is assumed that there is no direct link between group $\mathrm{S}$ and group D due to deep shadowing or blockage. Thus, the information exchange between $\mathrm{S}$ and $\mathrm{D}$ relies only on $R$. The non-orthogonally scheduled users form a NOMA group in the same channel, and each group works on a channel that is orthogonal to the channels used by the other groups [34]. We have assumed that $\mathrm{R}$ is a rather power-constrained node that acts as a half-duplex DF relay.

We have also assumed that channel state information is perfectly known and that each node in group $S$ and $D$ is a transceiver system that is equipped with a single antenna operating in a half-duplex mode. All the wireless channels are subject to the independent Rayleigh block fading plus additive white Gaussian noise with mean power $N_{0}$ in which the channel remains constant during the transmission of a block and varies independently from one block to another. We have also assumed that each user in group $\mathrm{S}$ and $\mathrm{D}$ are ordered according to their channel quality, i.e. $\left|h_{1}\right|^{2}>\left|h_{2}\right|^{2}>$ $\left|h_{3}\right|^{2}>\ldots . .\left|h_{N}\right|^{2}$ in group $S$ and $\left|g_{1}\right|^{2}<\left|g_{2}\right|^{2}<\left|g_{3}\right|^{2}<$ $\ldots . .\left|g_{N}\right|^{2}$ in group $D$. Here, $h_{1}, h_{2}, h_{3} \ldots h_{N}$ are denoted as complex channel coefficients of the $S_{1} \leftrightarrow R, S_{2} \leftrightarrow R$, $S_{3} \leftrightarrow R, \ldots, S_{N} \leftrightarrow R$ links, respectively, in group $S$ where $h_{i} \sim C N\left(0, \lambda_{h_{i}}=d_{i}^{-v}\right), d_{i}$ is the distance between $R$ and $i^{t h}$ user in group $S, \lambda_{h_{i}}$ is the variance and $v$ is the path loss exponent, $i=1,2, \ldots N$. Similarly, $g_{1}, g_{2}, g_{3} \ldots g_{N}$ are denoted as complex channel coefficients of the links, $D_{1} \leftrightarrow R$, $D_{2} \leftrightarrow R, D_{3} \leftrightarrow R, \ldots, D_{N} \leftrightarrow R$, respectively, in group $D$ where $g_{i} \sim C N\left(0, \lambda_{g_{i}}=d_{i}^{-v}\right)$. We have also assumed that the channels from the NOMA user nodes in group $S$ and $D$ to R and the channels from $\mathrm{R}$ to the NOMA user nodes in group $S$ and $D$ have the same fading impact. This means that the channels are reciprocal. The link gain or channel power gains are assumed to be exponential random variables (RV), whose cumulative distributive function (CDF) and probability density function (PDF) are given respectively as:

$$
\begin{aligned}
F_{\gamma}(x) & =1-e^{-\lambda_{i} x}, \\
f_{\gamma}(x) & =\lambda_{i} e^{-\lambda_{i} x},
\end{aligned}
$$

where $\lambda_{i}$ is the parameter of the channel gain between any two nodes.

\section{System Model Based on Time Switching ENERgy HARVESTING ARCHITECTURE}

For simplicity and without the loss of generality, we assume that there are two NOMA users in each group $\mathrm{S}$ and D i.e., $S_{1}$ and $S_{2}$ are NOMA users in group $\mathrm{S}$, and $D_{1}$ and $D_{2}$ are NOMA users in group D. Both group S and D works on a channel that is orthogonal to each other. The proposed system model scenario is depicted in Fig. 2. The two-user model is widely considered and investigated for NOMA systems [33][34]. Also, a two-user special case of NOMA, namely the multi-user superposition transmission (MUST), has been adopted by the Third Generation Partnership Project (3GPP) Long Term Evolution (LTE) [35]. The pairs of NOMA users in group $\mathrm{S}$ is ordered according to their channel gain such that $h_{2}<h_{1}$. Similarly, the pairs of NOMA users in group D is also ordered according to their channel gain such that $g_{1}<g_{2}$. Since, there are two users in each group, the total bandwidth $\mathrm{B}$ is equally divided between the group $\mathrm{S}$ and $\mathrm{D}$ based on proportional bandwidth fairness criterion, i.e. $B_{S}=B_{D}=\frac{B}{2}$. For simplicity, we have assumed $\mathrm{B}=1$.

Now, we explain our system model based on the time switching energy harvesting architecture [36], where the protocol is divided into two stages as shown in Fig. 3.

\section{A. First Stage}

In this stage, each NOMA user in group S, i.e. $S_{1}$ and $S_{2}$, and in group D, i.e. $D_{1}$ and $D_{2}$, non-orthogonally transmit the information signals to R using the uplink NOMA protocol. It should be noted that in an experiment conducted in Bristol, United Kingdom (UK), it was observed that cellular uplink 


\section{$\mathbf{T}$}

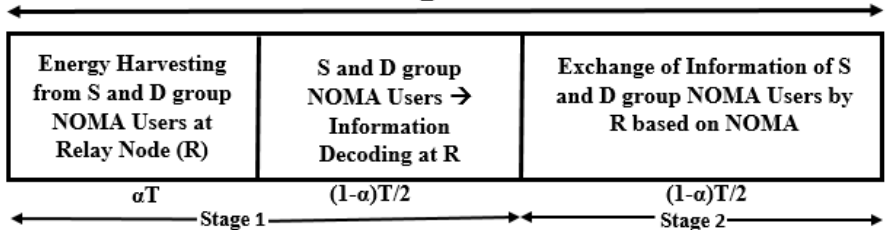

Fig. 3. BR NOMA-SWIPT based on the Time Switching protocol

could be a stable and strong source of RF energy, and significant energy available in the uplink could be harvested due to the high density of active user equipment (UEs) [37]. Following the uplink NOMA protocol, R successively decodes and cancels out the signals of strong channel users, prior to decoding the signals of weak channel users in-order to enhance the throughput of weak channel users. Since R is working as a DF time switching EH based relay, it uses the factor $\alpha$ to divide the total time block $T$ into three parts: $\alpha T, \frac{(1-\alpha) T}{2}$, and $\frac{(1-\alpha) T}{2}$ where $0 \leq \alpha \leq 1$. Here, in stage 1 , R first uses the $\alpha T$ period of time for RF energy harvesting from the information signal from the NOMA users in group $\mathrm{S}$ and $\mathrm{D}$, and then uses the $\frac{(1-\alpha) T}{2}$ time period for information processing as shown in Fig. 3. The information signal received at $\mathrm{R}$ during this stage is given by:

$$
\begin{gathered}
y_{R}=\sqrt{a_{1} P_{T}} h_{1} x_{S_{1}}+\sqrt{a_{2} P_{T}} h_{2} x_{S_{2}}+ \\
\sqrt{b_{1} P_{T}} g_{1} x_{D_{1}}+\sqrt{b_{2} P_{T}} g_{2} x_{D_{2}}+n_{R},
\end{gathered}
$$

where $x_{S_{1}}$, and $x_{S_{2}}$ are the information signals of the users $S_{1}$, and $S_{2}$ while $x_{D_{1}}$, and $x_{D_{2}}$ are the information signals of the users $D_{1}$, and $D_{2} . P_{T}$ is the transmission power at the NOMA user nodes and $n_{R} \sim C N\left(0, \sigma^{2}\right)$ is the additive white Gaussian noise at $\mathrm{R}$ with mean zero and variance $\sigma^{2}$. Furthermore, $a_{1}$, and $a_{2}$ with $a_{1}+a_{2}=1$, are the power allocation coefficients of $S_{1}$ and $S_{2}$ respectively whereas $b_{1}$, and $b_{2}$ with $b_{1}+b_{2}=1$, are the power allocation coefficients of $D_{1}$ and $D_{2}$, respectively.

The energy harvested at $\mathrm{R}$ in the $\alpha T$ time interval is given as:

$$
E_{R}=\eta \alpha T P_{T}\left(a_{1}\left|h_{1}\right|^{2}+a_{2}\left|h_{2}\right|^{2}+b_{1}\left|g_{1}\right|^{2}+b_{2}\left|g_{2}\right|^{2}\right),
$$

where $0 \leq \eta \leq 1$ represents the energy conversion efficiency. Its value depends on the energy harvesting circuitry of the relay node R. Now, the transmit power of $\mathrm{R}$ in the $\frac{(1-\alpha) T}{2}$ period of time is given by:

$P_{R}=\frac{E_{R}}{\frac{(1-\alpha) T}{2}}=\frac{2 \eta \alpha P_{T}\left(a_{1}\left|h_{1}\right|^{2}+a_{2}\left|h_{2}\right|^{2}+b_{1}\left|g_{1}\right|^{2}+b_{2}\left|g_{2}\right|^{2}\right)}{(1-\alpha)}$

In the above Equation 4, the pre-processing power of the energy harvesting circuitry is assumed to be negligible in contrast to the transmission power $P_{R}$ of the R [38].

As stated before, by applying the uplink NOMA protocol, $\mathrm{R}$ first decodes the strong channel users, i.e. $S_{1}$ from group $\mathrm{S}$ and $D_{2}$ from group $\mathrm{D}$, by treating $S_{2}$ from group $\mathrm{S}$ and $D_{1}$ from group $\mathrm{D}$ as residual interference signal. $\mathrm{R}$ then applies SIC before decoding the information signal of $S_{2}$ from group $\mathrm{S}$ and $D_{1}$ from group $\mathrm{D}$. Therefore, the received signal-to- interference-plus-noise ratios (SINR) at $\mathrm{R}$ of the $R \rightarrow S_{1}$ link and the $R \rightarrow S_{2}$ link of group $\mathrm{S}$ are given by:

$$
\begin{gathered}
\gamma_{R \rightarrow S_{1}}=\frac{\rho a_{1}\left|h_{1}\right|^{2}}{\rho a_{2}\left|h_{2}\right|^{2}+1}, \\
\gamma_{R \rightarrow S_{2}}=\frac{\rho a_{2}\left|h_{2}\right|^{2}}{\rho a_{1}\left|\hat{h_{1}}\right|^{2}+1},
\end{gathered}
$$

where $\hat{h_{1}} \sim C N\left(0, \xi \lambda_{h_{1}}\right)$ and $\rho=\frac{P_{T}}{N_{0}}$ represents the transmit signal-to-noise ratio (SNR). The parameter $\xi, 0 \leq \xi \leq 1$ denotes the residual interference signal caused by the SIC imperfection at $\mathrm{R}$, where $\xi=0$ denotes the perfect SIC (pSIC) and $\xi=1$ denotes the imperfect SIC (ipSIC) employed at R.

Similarly, the received SINR values of the $R \rightarrow D_{2}$ link and of the $R \rightarrow D_{1}$ link of group $\mathrm{D}$ are given by:

$$
\begin{gathered}
\gamma_{R \rightarrow D_{2}}=\frac{\rho b_{2}\left|g_{2}\right|^{2}}{\rho b_{1}\left|g_{1}\right|^{2}+1}, \\
\gamma_{R \rightarrow D_{1}}=\frac{\rho b_{1}\left|g_{1}\right|^{2}}{\rho b_{2}\left|\hat{g_{2}}\right|^{2}+1},
\end{gathered}
$$

where $\hat{g_{2}} \sim C N\left(0, \xi \lambda_{g_{2}}\right)$.

\section{B. Second Stage}

In the second stage, the information between the two NOMA group users is exchanged with the help $\mathrm{R}$ using the downlink NOMA protocol. In this protocol, the strong channel NOMA users successively decodes and cancels the signals of the weak channel NOMA users, prior to decoding their own signal. Accordingly, following the downlink NOMA protocol, $\mathrm{R}$ transmits a superimposed composite signals $\left(\sqrt{a_{3} P_{R}} x_{S_{1}}+\right.$ $\left.\sqrt{a_{4} P_{R}} x_{S_{2}}\right)$ and $\left(\sqrt{b_{3} P_{R}} x_{D_{1}}+\sqrt{b_{4} P_{R}} x_{D_{2}}\right)$ to group $S$ and $D$, respectively, using the same orthogonal channel, as we have assumed channel reciprocity. Here, $P_{R}$ is the total transmit power of the $\mathrm{R}$, and $a_{3}$, and $a_{4}$ are the power allocation coefficients of $S_{1}$ and $S_{2}$, respectively, whereas $b_{3}$, and $b_{4}$ are the power allocation coefficients of $D_{1}$ and $D_{2}$, respectively. Since, we have used downlink NOMA in this stage, the total power transmit power constraint of $\mathrm{R}$ for the users in the $\mathrm{S}$ and $\mathrm{D}$ groups implies that $a_{3}<a_{4}$ and $b_{3}>b_{4}$ with $a_{3}+a_{4}+b_{3}+b_{4}=1$.

The received SINR values of the $S_{1} \rightarrow D_{2}$ link, $S_{1} \rightarrow D_{1}$ link and $S_{2} \rightarrow D_{2}$ link during the second stage stage are given by:

$$
\begin{aligned}
& \gamma_{S_{1} \rightarrow D_{2}}=\frac{b_{4} P_{R}\left|h_{1}\right|^{2}}{b_{3} P_{R}\left|h_{1}\right|^{2}+1} \\
& \gamma_{S_{1} \rightarrow D_{1}}=\frac{b_{3} P_{R}\left|h_{1}\right|^{2}}{b_{4} P_{R}\left|\hat{h_{1}}\right|^{2}+1} \\
& \gamma_{S_{2} \rightarrow D_{2}}=\frac{b_{4} P_{R}\left|h_{2}\right|^{2}}{b_{3} P_{R}\left|h_{2}\right|^{2}+1}
\end{aligned}
$$

Similarly, the received SINR values of the $D_{2} \rightarrow S_{1}$ link, $D_{2} \rightarrow S_{2}$ link and $D_{1} \rightarrow S_{1}$ link during this stage are given by:

$$
\gamma_{D_{2} \rightarrow S_{1}}=\frac{a_{3} P_{R}\left|g_{2}\right|^{2}}{a_{4} P_{R}\left|g_{2}\right|^{2}+1}
$$




$$
\begin{gathered}
\gamma_{D_{2} \rightarrow S_{2}}=\frac{a_{4} P_{R}\left|g_{2}\right|^{2}}{a_{3} P_{R}\left|\hat{g_{2}}\right|^{2}+1} \\
\gamma_{D_{1} \rightarrow S_{1}}=\frac{a_{3} P_{R}\left|g_{1}\right|^{2}}{a_{4} P_{R}\left|g_{1}\right|^{2}+1}
\end{gathered}
$$

From the above two stages, the exchange of information between the two groups $\mathrm{S}$ and $\mathrm{D}$ is finally accomplished with the assistance of R. Specifically, the signal $x_{S_{1}}$ of $S_{1}$ is exchanged together with the signal $x_{D_{1}}$ of $D_{1}$. Furthermore, the signal $x_{S_{2}}$ of $S_{2}$ is exchanged together with the signal $x_{D_{2}}$ of $\mathrm{D}_{2}$.

\section{Outage Probability Analysis}

In this section, we provide analytical derivations of the outage probability for our BR NOMA-SWIPT system. We will investigate the outage probability of each of the nodes, i.e. of $S_{1}$, and $S_{2}$ in group $S$ and of $D_{1}$, and $D_{2}$ in group $D$.

\section{A. Outage Probability of $S_{1}$}

The $S_{1}$ NOMA user will be in outage if any of the following conditions hold true:

1) $\mathrm{R}$ fails to decode the transmitted signal from $S_{1}$ in the first stage.

2) $D_{1}$ cannot decode the signal transmitted from $\mathrm{R}$ in the second stage.

3) $D_{2}$ cannot decode the signal transmitted from $\mathrm{R}$ in the second stage.

The above three conditions for the outage probability of $S_{1}$, can be expressed as:

$$
P_{S_{1}}^{\text {Out }}=1-\operatorname{Pr}\left(\gamma_{R \rightarrow S_{1}}>\gamma_{S_{1}}^{T}, \gamma_{D_{1} \rightarrow S_{1}}>\gamma_{D_{1}}^{T}, \gamma_{D_{2} \rightarrow S_{1}}>\gamma_{D_{2}}^{T}\right)
$$

where $\gamma_{S_{1}}^{T}=2^{2 R_{S_{1}}}-1$ is the lower SNR threshold value i.e., the outage probability of $S_{1}$ with $R_{S_{1}}$ denoting the target data rate of $S_{1} ; \gamma_{D_{1}}^{T}=2^{2 R_{D_{1}}}-1$ is the lower SNR threshold value of $D_{1}$ with $R_{D_{1}}$ denoting the target data rate of the $D_{1}$, and $\gamma_{D_{2}}^{T}=2^{2 R_{D_{2}}}-1$ is the lower threshold value for SNR of $D_{2}$ with $R_{D_{2}}$ as the target data rate of $D_{2}$.

The following theorem provides the outage probability of $S_{1}$ for the BR NOMA-SWIPT system:

Theorem 1. The closed-form expression for the outage probability of the $S_{1}$ NOMA user for BR NOMA with SWIPT can be expressed as in Equation 18:
Proof: The detailed proof is given in Appendix A.

\section{B. Outage Probability of $S_{2}$}

The $S_{2}$ NOMA user will be in outage if any of the following conditions hold true:

1) $\mathrm{R}$ fails to decode the transmitted signal from $S_{2}$ in the first stage.

2) $D_{2}$ cannot decode the transmitted signal of $S_{2}$ from $\mathrm{R}$ in the second stage.

The above two conditions of the outage probability of $S_{2}$ can be expressed as:

$$
P_{S_{2}}^{\text {Out }}=1-\operatorname{Pr}\left(\gamma_{R \rightarrow S_{2}}>\gamma_{S_{2}}^{T}, \gamma_{D_{2} \rightarrow S_{2}}>\gamma_{D_{2}}^{T}\right)
$$

where $\gamma_{S_{2}}^{T}=2^{2 R_{S_{2}}}-1$ is the lower SNR threshold value of $S_{2}$ with $R_{S_{2}}$ denoting the target data rate of $S_{2}$.

The following theorem provides the outage probability of $S_{2}$ for the BR NOMA-SWIPT system:

Theorem 2. The closed-form expression for the outage probability of the $S_{2}$ NOMA user for BR NOMA with SWIPT can be expressed as in Equation 19:

Proof: The detailed proof is given in Appendix B.

\section{Outage Probability of $D_{1}$}

The $D_{1}$ NOMA user will be in outage if any of the following conditions hold true:

1) $\mathrm{R}$ fails to decode the transmitted signal from $D_{1}$ in the first stage.

2) $S_{1}$ cannot decode the transmitted signal of $S_{2}$ from $\mathrm{R}$ in the second stage.

The above two conditions of the outage probability of $D_{1}$ can be expressed as:

$$
P_{D_{1}}^{\text {Out }}=1-\operatorname{Pr}\left(\gamma_{R \rightarrow D_{1}}>\gamma_{D_{1}}^{T}, \gamma_{S_{1} \rightarrow D_{1}}>\gamma_{S_{1}}^{T}\right)
$$

The following theorem provides the outage probability of $D_{1}$ for the BR NOMA-SWIPT system:

Theorem 3. The closed-form expression for the outage probability of the D $D_{1}$ NOMA user for BR NOMA with SWIPT can be expressed as in Equation 20:

Proof: The proof can be derived by following the similar steps as in Appendix B.

$$
\begin{aligned}
& P_{S_{1}}^{\text {Out }}=1-\left(A_{1} \sum_{n=0}^{\infty} \frac{(-1)^{n}}{n !}\left(\lambda_{g_{2}}-\lambda_{h_{1}}+\left(\lambda_{g_{1}}-\lambda_{h_{1}}\right) \frac{z_{1}}{z_{2}}\right)^{n}\left(y_{2}^{g}\right)^{n+1} E_{n+2}\left(\frac{\lambda_{h_{1}} z_{2}}{y_{2}^{g}}\right)-B_{1} \sum_{n=0}^{\infty} \frac{(-1)^{n}}{n !}\left(\lambda_{g_{2}}-\eta_{1}+\left(\lambda_{g_{1}}-\eta_{1}\right) \frac{z_{1}}{z_{2}}\right)^{n}\left(y_{2}^{g}\right)^{n+1}\right. \\
& E_{n+2}\left(\frac{\eta_{1} z_{2}}{y_{2}^{g}}\right)+C_{1} \sum_{n=0}^{\infty} \frac{(-1)^{n}}{n !}\left(\lambda_{g_{2}}-\lambda_{g_{1}}\right)^{n}\left(y_{2}^{g}\right)^{n+1} E_{n+2}\left(\frac{\lambda_{g_{1}} z_{2}}{y_{2}^{g}}\right)+D_{1}+A_{2} \sum_{n=0}^{\infty} \frac{(-1)^{n}}{n !}\left(\lambda_{g_{1}}-\lambda_{h_{1}}+\left(\lambda_{g_{2}}-\lambda_{h_{1}}\right) \frac{z_{2}}{z_{1}}\right)^{n}\left(y_{1}^{g}\right)^{n+1} E_{n+2}\left(\frac{\lambda_{h_{1}} z_{1}}{y_{1}^{g}}\right)- \\
&\left.B_{2} \sum_{n=0}^{\infty} \frac{(-1)^{n}}{n !}\left(\lambda_{g_{1}}-\eta_{1}+\left(\lambda_{g_{2}}-\eta_{1}\right) \frac{z_{2}}{z_{1}}\right)^{n}\left(y_{1}^{g}\right)^{n+1} E_{n+2}\left(\frac{\eta_{1} z_{1}}{y_{1}^{g}}\right)+C_{2} \sum_{n=0}^{\infty} \frac{(-1)^{n}}{n !}\left(\lambda_{g_{1}}-\lambda_{g_{2}}\right)^{n}\left(y_{1}^{g}\right)^{n+1} E_{n+2}\left(\frac{\lambda_{g_{2}} z_{1}}{y_{1}^{g}}\right)+D_{2}\right)
\end{aligned}
$$


where $c=\frac{2 \eta \alpha P_{T}}{(1-\alpha)}, t=\frac{\gamma}{\rho}, z_{1}=\frac{b_{1} \gamma}{c\left(a_{3}-a_{4} \gamma\right)}, z_{2}=\frac{b_{2} \gamma}{c\left(a_{3}-a_{4} \gamma\right)}$,

$\eta_{1}=\frac{\lambda_{h_{2}}+\gamma \lambda_{h_{1}}}{1+\gamma}, \delta=\frac{t}{1+\gamma}, y_{1}^{g}=\frac{z_{1}}{z_{1}+z_{2}}\left(\sqrt{\left(z_{1}+z_{2}\right)+\frac{t^{2}}{4}}-\frac{t}{2}\right), y_{2}^{g}=\frac{z_{2}}{z_{1}+z_{2}}\left(\sqrt{\left(z_{1}+z_{2}\right)+\frac{t^{2}}{4}}-\frac{t}{2}\right)$,

$A_{1}=\frac{\lambda_{h_{2}} \lambda_{g_{1}} \lambda_{g_{2}}}{\left(\lambda_{h_{1}}-\lambda_{h_{2}}\right)\left(\lambda_{h_{1}}-\lambda_{g_{1}}\right)}, B_{1}=\frac{\lambda_{h_{1}} \lambda_{h_{2}} \lambda_{g_{1}} \lambda_{g_{2}} e^{-\left(\lambda_{h_{1}}-\lambda_{h_{2}}\right) \delta}}{\eta_{1}\left(\lambda_{h_{1}}-\lambda_{h_{2}}\right)\left(\eta_{1}-\lambda_{g_{1}}\right)}, C_{1}=\frac{\lambda_{h_{1}} \lambda_{h_{2}} \lambda_{g_{2}} e^{-\left(\lambda_{h_{1}}-\lambda g_{1}\right) t}}{(1+\gamma)\left(\eta_{1}-\lambda_{g_{1}}\right)\left(\lambda_{h_{1}}-\lambda_{g_{1}}\right)}, D_{1}=\frac{\lambda_{h_{2}} \lambda_{g_{2}} e^{-\lambda_{h_{1}} t-\left(\lambda_{g_{1}} z_{1}+z_{g_{2}}\right) y_{2}^{g}}}{\left(\lambda_{g_{2}}+\gamma \lambda_{h_{1}}\right)\left(\lambda_{g_{1}} \frac{z_{1}}{z_{2}}+\lambda_{g_{2}}\right)}$,

$A_{2}=\frac{\lambda_{h_{2}} \lambda_{g_{1}} \lambda_{g_{2}}}{\left(\lambda_{h_{1}}-\lambda_{h_{2}}\right)\left(\lambda_{h_{1}}-\lambda_{g_{2}}\right)}, B_{2}=\frac{\lambda_{h_{1}} \lambda_{h_{2}} \lambda_{g_{1}} \lambda_{g_{2}} e^{-\left(\lambda_{h_{1}}-\lambda_{h_{2}}\right) \delta}}{\eta_{1}\left(\lambda_{h_{1}}-\lambda_{h_{2}}\right)\left(\eta_{1}-\lambda_{g_{2}}\right)}, C_{2}=\frac{\lambda_{h_{1}} \lambda_{h_{2}} \lambda_{g_{2}} e^{-\left(\lambda_{h_{1}}-\lambda g_{2}\right) t}}{(1+\gamma)\left(\eta_{1}-\lambda_{g_{2}}\right)\left(\lambda_{h_{1}}-\lambda_{g_{2}}\right)}, D_{2}=\frac{\lambda_{h_{2}} \lambda_{g_{1}} e^{-\lambda_{h_{1}} t-\left(\lambda_{g_{2}} \frac{z_{1}}{\left.z_{2}+\lambda g_{1}\right) y_{1}^{g}}\right.}}{\left(\lambda_{g_{2}}+\gamma \lambda_{h_{1}}\right)\left(\lambda_{g_{2}} \frac{z_{2}}{z_{1}}+\lambda_{g_{1}}\right)}$ and $E_{n+2}(a)=\int_{y=1}^{\infty} y^{-n-2} e^{-a y} d y$ is an exponential integral of order $\mathrm{n}+2$.

$$
\begin{aligned}
P_{S_{2}}^{\text {Out }}=1- & \left(A_{S_{2}} \lambda_{g_{2}} \sum_{n=0}^{\infty} \frac{(-1)^{n}}{n !}\left(\lambda_{g_{2}}+\lambda_{h_{2}}\right)^{n}\left(y_{2}^{*}\right)^{n+1} E_{n+2}\left(\frac{\lambda_{h_{2}} t}{y_{2}^{*}}\right)+B_{S_{2}} \lambda_{g_{2}} \sum_{n=0}^{\infty} \frac{(-1)^{n}}{n !}\left(\lambda_{g_{2}}-\lambda_{g_{1}}\right)^{n}\left(y_{2}^{*}\right)^{n+1} E_{n+2}\left(\frac{\lambda_{g_{1}} t}{y_{2}^{*}}\right)+\right. \\
& \left.C_{S_{2}} \lambda_{g_{2}} \sum_{n=0}^{\infty} \frac{(-1)^{n}}{n !}\left(\frac{\left(\lambda_{g_{2}}-\lambda_{h_{1}}\right)+\left(\lambda_{g_{2}}-\lambda_{h_{2}}\right) \xi \gamma}{(1+\xi \gamma)}\right)^{n}\left(y_{2}^{*}\right)^{n+1} E_{n+2}\left(\frac{\left(\lambda_{h_{1}}+\xi \gamma \lambda_{h_{2}}\right) t}{(1+\xi \gamma) y_{2}^{*}}\right)+\frac{\lambda_{h_{1}} e^{-k \lambda_{h_{2}}}}{\xi \gamma \lambda_{h_{2}}+\lambda_{h_{1}}} e^{-\lambda_{g_{2}} y_{2}^{*}}\right)
\end{aligned}
$$

where $c=\frac{2 \eta \alpha P_{T}}{(1-\alpha)}, k=\frac{\gamma}{\rho}, d_{1}=\frac{a_{4}}{b_{2}} c, d_{2}=\frac{a_{3} \xi c}{b_{2}}, t=\frac{\gamma}{d_{1}-\gamma d_{2}}, y_{2}^{*}=\frac{-k+\sqrt{k^{2}+4 t}}{2}, A_{S_{2}}=\frac{\lambda_{h_{1}} \lambda_{g_{1}}}{\left(\lambda_{h_{1}}-\lambda_{h_{2}}\right)\left(\lambda_{g_{1}}-\lambda_{h_{2}}\right)}, B_{S_{2}}=\frac{\lambda_{h_{1}} e^{k\left(\lambda_{g_{1}}-\lambda_{h_{2}}\right)}}{\xi \gamma \lambda_{h_{2}}+\lambda_{h_{1}}}-$ $\frac{\lambda_{h_{1}} \lambda_{g_{1}} e^{\left(\lambda_{g_{1}}-\lambda_{h_{2}}\right) k}}{\left(\lambda_{h_{1}}-\lambda_{h_{2}}\right)\left(\lambda_{g_{1}}-\lambda_{h_{2}}\right)}-\left(\frac{\lambda_{h_{1}} \lambda_{g_{1}}}{\left(\lambda_{h_{1}}+\xi \gamma \lambda_{h_{2}}\right)}-\frac{\lambda_{h_{1}} \lambda_{g_{1}}}{\left(\lambda_{h_{1}}-\lambda_{h_{2}}\right)}\right) \frac{(1+\xi \gamma) e^{\left(\lambda_{g_{1}}-\lambda_{h_{2}}\right) k}}{\left(\lambda_{g_{1}}-\lambda_{h_{1}}\right)+\left(\lambda_{g_{1}}-\lambda_{h_{2}}\right) \xi \gamma}, C_{S_{2}}=\left(\frac{\lambda_{h_{1}} \lambda_{g_{1}}}{\left(\lambda_{h_{1}}+\xi \gamma \lambda_{h_{2}}\right)}-\frac{\lambda_{h_{1}} \lambda_{g_{1}}}{\left(\lambda_{h_{1}}-\lambda_{h_{2}}\right)}\right) \frac{e^{\frac{k\left(\lambda_{h_{1}}-\lambda_{h_{2}}\right)}{(1+\xi \gamma)}}(1+\xi \gamma)}{\left(\lambda_{g_{1}}-\lambda_{h_{1}}\right)+\left(\lambda_{g_{1}}-\lambda_{h_{2}}\right) \xi \gamma}$

$$
\begin{array}{r}
P_{D_{1}}^{\text {Out }}=1-\left(A_{D_{2}} \lambda_{h_{1}} \sum_{n=0}^{\infty} \frac{(-1)^{n}}{n !}\left(\lambda_{h_{1}}+\lambda_{g_{1}}\right)^{n}\left(x_{1}^{*}\right)^{n+1} E_{n+2}\left(\frac{\lambda_{g_{1}} t}{x_{1}^{*}}\right)+B_{D_{1}} \lambda_{h_{1}} \sum_{n=0}^{\infty} \frac{(-1)^{n}}{n !}\left(\lambda_{h_{1}}-\lambda_{h_{2}}\right)^{n}\left(x_{1}^{*}\right)^{n+1} E_{n+2}\left(\frac{\lambda_{h_{2}} t}{x_{1}^{*}}\right)+\right. \\
\left.C_{D_{1}} \lambda_{h_{1}} \sum_{n=0}^{\infty} \frac{(-1)^{n}}{n !}\left(\frac{\left(\lambda_{h_{1}}-\lambda_{g_{2}}\right)+\left(\lambda_{h_{1}}-\lambda_{g_{1}}\right) \xi \gamma}{(1+\xi \gamma)}\right)^{n}\left(x_{1}^{*}\right)^{n+1} E_{n+2}\left(\frac{\left(\lambda_{g_{2}}+\xi \gamma \lambda_{g_{1}}\right) t}{(1+\xi \gamma) x_{1}^{*}}\right)+\frac{\lambda_{g_{2}} e^{-k \lambda_{g_{1}}}}{\xi \gamma \lambda_{g_{1}}+\lambda_{g_{2}}} e^{-\lambda_{h_{1}} x_{1}^{*}}\right)
\end{array}
$$

where $c=\frac{2 \eta \alpha P_{T}}{(1-\alpha)}, k=\frac{\gamma}{\rho}, d_{1}=\frac{b_{3}}{a_{1}} c, d_{2}=\frac{b_{4} \xi c}{a_{1}}, t=\frac{\gamma}{d_{1}-\gamma d_{2}}, x_{1}^{*}=\frac{-k+\sqrt{k^{2}+4 t}}{2}, A_{D_{1}}=\frac{\lambda_{g_{2}} \lambda_{h_{2}}}{\left(\lambda_{g_{2}}-\lambda_{g_{1}}\right)\left(\lambda_{h_{2}}-\lambda_{g_{1}}\right)}, B_{D_{1}}=\frac{\lambda_{g_{2}} e^{k\left(\lambda_{h_{2}}-\lambda_{g_{1}}\right)}}{\xi \gamma \lambda_{g_{1}}+\lambda_{g_{2}}}-$ $\frac{\lambda_{g_{2}} \lambda_{h_{2}} e^{\left(\lambda_{h_{2}}-\lambda_{g_{1}}\right) k}}{\left(\lambda_{g_{2}}-\lambda_{g_{1}}\right)\left(\lambda_{h_{2}}-\lambda_{g_{1}}\right)}-\left(\frac{\lambda_{g_{2}} \lambda_{h_{2}}}{\left(\lambda_{g_{2}}+\xi \gamma \lambda_{g_{1}}\right)}-\frac{\lambda_{g_{2}} \lambda_{h_{2}}}{\left(\lambda_{g_{2}}-\lambda_{g_{1}}\right)}\right) \frac{(1+\xi \gamma) e^{\left(\lambda_{h_{2}}-\lambda_{g_{1}}\right) k}}{\left(\lambda_{h_{2}}-\lambda_{g_{2}}\right)+\left(\lambda_{h_{2}}-\lambda_{g_{1}}\right) \xi \gamma}, C_{D_{1}}=\left(\frac{\lambda_{g_{2}} \lambda_{h_{2}}}{\left(\lambda_{g_{2}}+\xi \gamma \lambda_{g_{1}}\right)}-\frac{\lambda_{g_{2}} \lambda_{h_{2}}}{\left(\lambda_{g_{2}}-\lambda_{g_{1}}\right)}\right) \frac{e^{\frac{k\left(\lambda_{g_{2}}-\lambda g_{1}\right)}{(1+\xi \gamma)}}(1+\xi \gamma)}{\left(\lambda_{h_{2}}-\lambda_{g_{2}}\right)+\left(\lambda_{h_{2}}-\lambda_{g_{1}}\right) \xi \gamma}$

$$
\begin{aligned}
& P_{D_{2}}^{\text {Out }}=1-\left(A_{3} \sum_{n=0}^{\infty} \frac{(-1)^{n}}{n !}\left(\lambda_{h_{1}}-\lambda_{g_{2}}+\left(\lambda_{h_{2}}-\lambda_{g_{2}}\right) \frac{z_{1}}{z_{2}}\right)^{n}\left(x_{1}^{g}\right)^{n+1} E_{n+2}\left(\frac{\lambda_{g_{2}} z_{2}}{x_{1}^{g}}\right)-B_{3} \sum_{n=0}^{\infty} \frac{(-1)^{n}}{n !}\left(\lambda_{h_{1}}-\eta_{1}+\left(\lambda_{h_{2}}-\eta_{1}\right) \frac{z_{1}}{z_{2}}\right)^{n}\left(x_{1}^{g}\right)^{n+1}\right. \\
& E_{n+2}\left(\frac{\eta_{1} z_{2}}{x_{1}^{g}}\right)+C_{3} \sum_{n=0}^{\infty} \frac{(-1)^{n}}{n !}\left(\lambda_{h_{1}}-\lambda_{h_{2}}\right)^{n}\left(x_{1}^{g}\right)^{n+1} E_{n+2}\left(\frac{\lambda_{h_{2}} z_{2}}{x_{1}^{g}}\right)+D_{3}+A_{4} \sum_{n=0}^{\infty} \frac{(-1)^{n}}{n !}\left(\lambda_{h_{2}}-\lambda_{g_{2}}+\left(\lambda_{h_{1}}-\lambda_{g_{2}}\right) \frac{z_{2}}{z_{1}}\right)^{n}\left(x_{2}^{g}\right)^{n+1} E_{n+2}\left(\frac{\lambda_{g_{2}} z_{1}}{x_{2}^{g}}\right)- \\
&\left.B_{4} \sum_{n=0}^{\infty} \frac{(-1)^{n}}{n !}\left(\lambda_{h_{2}}-\eta_{1}+\left(\lambda_{h_{1}}-\eta_{1}\right) \frac{z_{2}}{z_{1}}\right)^{n}\left(x_{2}^{g}\right)^{n+1} E_{n+2}\left(\frac{\eta_{1} z_{1}}{x_{2}^{g}}\right)+C_{4} \sum_{n=0}^{\infty} \frac{(-1)^{n}}{n !}\left(\lambda_{h_{2}}-\lambda_{h_{1}}\right)^{n}\left(x_{2}^{g}\right)^{n+1} E_{n+2}\left(\frac{\lambda_{h_{1}} z_{1}}{x_{2}^{g}}\right)+D_{4}\right)
\end{aligned}
$$

where $c=\frac{2 \eta \alpha P_{T}}{(1-\alpha)}, t=\frac{\gamma}{\rho}, z_{1}=\frac{a_{2} \gamma}{c\left(b_{4}-b_{3} \gamma\right)}, z_{2}=\frac{a_{1} \gamma}{c\left(b_{4}-b_{3} \gamma\right)}$,

$\eta_{1}=\frac{\lambda_{g_{1}}+\gamma \lambda_{g_{2}}}{1+\gamma}, \delta=\frac{t}{1+\gamma}, x_{2}^{g}=\frac{z_{1}}{z_{1}+z_{2}}\left(\sqrt{\left(z_{1}+z_{2}\right)+\frac{t^{2}}{4}}-\frac{t}{2}\right), x_{1}^{g}=\frac{z_{2}}{z_{1}+z_{2}}\left(\sqrt{\left(z_{1}+z_{2}\right)+\frac{t^{2}}{4}}-\frac{t}{2}\right)$,

$A_{3}=\frac{\lambda_{g_{1}} \lambda_{h_{2}} \lambda_{h_{1}}}{\left(\lambda_{g_{2}}-\lambda_{g_{1}}\right)\left(\lambda_{g_{2}}-\lambda_{h_{2}}\right)}, B_{3}=\frac{\lambda_{g_{2}} \lambda_{g_{1}} \lambda_{h_{2}} \lambda_{h_{1}} e^{-\left(\lambda g_{2}-\lambda g_{1}\right) \delta}}{\eta_{1}\left(\lambda_{g_{2}}-\lambda_{g_{1}}\right)\left(\eta_{1}-\lambda_{h_{2}}\right)}, C_{3}=\frac{\lambda_{g_{2}} \lambda_{g_{1}} \lambda_{h_{1}} e^{-\left(\lambda_{g_{2}}-\lambda_{h_{2}}\right) t}}{(1+\gamma)\left(\eta_{1}-\lambda_{h_{2}}\right)\left(\lambda_{g_{2}}-\lambda_{h_{2}}\right)}, D_{3}=\frac{\lambda_{g_{1}} \lambda_{h_{1}} e^{-\lambda g_{2} t-\left(\lambda_{h_{2}} \frac{z_{1}}{z_{2}+\lambda h_{1}}\right) x_{1}^{g}}}{\left(\lambda_{h_{1}}+\gamma \lambda_{g_{2}}\right)\left(\lambda_{h_{2}} \frac{z_{1}}{z_{2}}+\lambda_{h_{1}}\right)}$,

$A_{4}=\frac{\lambda_{g_{1}} \lambda_{h_{2}} \lambda_{h_{1}}}{\left(\lambda_{g_{2}}-\lambda_{g_{1}}\right)\left(\lambda_{g_{2}}-\lambda_{h_{1}}\right)}, B_{4}=\frac{\lambda_{g_{2}} \lambda_{g_{1}} \lambda_{h_{2}} \lambda_{h_{1}} e^{-\left(\lambda g_{2}-\lambda g_{1}\right) \delta}}{\eta_{1}\left(\lambda_{g_{2}}-\lambda_{g_{1}}\right)\left(\eta_{1}-\lambda_{h_{1}}\right)}, C_{4}=\frac{\lambda_{g_{2}} \lambda_{g_{1}} \lambda_{h_{1}} e^{-\left(\lambda_{g_{2}}-\lambda_{h_{1}}\right) t}}{(1+\gamma)\left(\eta_{1}-\lambda_{h_{1}}\right)\left(\lambda_{g_{2}}-\lambda_{h_{1}}\right)}, D_{4}=\frac{\lambda_{g_{1}} \lambda_{h_{2}} e^{-\lambda_{g_{2}} t-\left(\lambda_{h_{1}} \frac{z_{1}}{z_{2}}+\lambda_{h_{2}}\right) x_{2}^{g}}}{\left(\lambda_{h_{1}}+\gamma \lambda_{g_{2}}\right)\left(\lambda_{h_{1}} \frac{z_{2}}{z_{1}}+\lambda_{h_{2}}\right)}$. 


\section{Outage Probability of $D_{2}$}

The $D_{2}$ NOMA user will be in outage if any of the following conditions hold true:

1) $\mathrm{R}$ fails to decode the transmitted signal from $D_{2}$ in the first stage.

2) $S_{2}$ cannot decode the signal transmitted from $\mathrm{R}$ in the second stage.

3) $S_{1}$ cannot decode the signal transmitted from $\mathrm{R}$ in the second stage.

The above three conditions of the outage probability of $D_{2}$, can be expressed as:

$P_{D_{2}}^{\text {Out }}=1-\operatorname{Pr}\left(\gamma_{R \rightarrow D_{2}}>\gamma_{D_{2}}^{T}, \gamma_{S_{2} \rightarrow D_{2}}>\gamma_{D_{2}}^{T}, \gamma_{S_{1} \rightarrow D_{2}}>\gamma_{S_{1}}^{T}\right)$

The following theorem provides the outage probability of $D_{2}$ for the BR NOMA-SWIPT system:

Theorem 4. The closed-form expression for the outage probability of the $D_{2}$ NOMA user for BR NOMA with SWIPT can be expressed as in Equation 21:

Proof: The proof can be derived by following the similar steps as in Appendix A.

\section{E. Sum-throughput of the BR NOMA SWIPT System - Delay Limited Capacity Analysis}

In delay-limited transmission mode, the system throughput is limited by a fixed rate. The overall throughput in our BR NOMA-SWIPT enabled IoT relay networks for the $S_{1}, D_{1}, S_{2}$ and $D_{2}$ NOMA users can be given as:

$$
T h r_{S y s}=\frac{\left(1-P_{S_{1}}^{\text {Out }}\right)(1-\alpha) R_{S_{1}}}{2}+\frac{\left(1-P_{D_{1}}^{\text {Out }}\right)(1-\alpha) R_{D_{1}}}{2}+
$$

Finally, substituting the outage probabilities of the individual NOMA users in Equation 23 with their analytical expressions given in Equations 16, 18, 20, and 22, we get the analytical expression for the overall sum-throughput of the proposed BR NOMA-SWIPT system.

\section{F. Ergodic Capacity and Ergodic Sum Capacity Analysis - Delay Tolerant Capacity Analysis}

In this section, we analyze the ergodic capacity and ergodic sum capacity analysis for our BR NOMA-SWIPT enabled IoT relay networks. We will first investigate the individual ergodic capacities of $S_{1}, S_{2}, D_{1}$ and $D_{2}$ and then finally the ergodic sum capacity of the proposed system.

According to our system model presented in the above section, the individual ergodic capacities of NOMA users are given by:

$$
\begin{gathered}
C_{S_{1}}=\frac{(1-\alpha)}{2} E\left[\log _{2}\left(1+\min \left(\gamma_{R \rightarrow S_{1}}, \gamma_{D_{1} \rightarrow S_{1}}, \gamma_{D_{2} \rightarrow S_{1}}\right)\right)\right] \\
C_{D_{1}}=\frac{(1-\alpha)}{2} E\left[\log _{2}\left(1+\min \left(\gamma_{R \rightarrow D_{1}}, \gamma_{S_{1} \rightarrow D_{1}}\right)\right)\right]
\end{gathered}
$$

$$
\begin{gathered}
C_{S_{2}}=\frac{(1-\alpha)}{2} E\left[\log _{2}\left(1+\min \left(\gamma_{R \rightarrow S_{2}}, \gamma_{D_{2} \rightarrow S_{2}}\right)\right)\right] \\
C_{D_{2}}=\frac{(1-\alpha)}{2} E\left[\log _{2}\left(1+\min \left(\gamma_{R \rightarrow D_{2}}, \gamma_{S_{2} \rightarrow D_{2}}, \gamma_{S_{1} \rightarrow D_{2}}\right)\right)\right]
\end{gathered}
$$

where $E[$.$] denotes the statistical expectation operator.$

The overall ergodic sum capacity of the proposed system is:

$$
C_{S y s}=C_{S_{1}}+C_{D_{1}}+C_{S_{2}}+C_{D_{2}}
$$

To derive the analytical expressions for the ergodic capacities of the individual NOMA users and the sum capacity of the proposed BR NOMA-SWIPT system, we make use of the ergodic capacity equation in terms of a $\mathrm{CDF}$, which can be written as [39]:

$$
C=\frac{(1-\alpha)}{2 \ln 2} \int_{0}^{\infty} \frac{1}{1+w}\left[1-F_{W}(w)\right] d w
$$

where $F_{W}(w)$ is the CDF of a random variable of w. The $\mathrm{CDF}$ of the minimum of the random variables for $S_{1}, D_{1}$, $S_{2}$ and $D_{2}$, as given by the ergodic capacity Equations 24, 25,26 and 27, can be found by following the same steps as in Appendix A and Appendix B. For the sake of brevity, we have omitted the detailed steps here. Finally, after finding the CDF for each NOMA user and substituting into Equation 29, we get the analytical expression for the ergodic capacity of each individual NOMA user. However, due to the involvement of the complex terms in the CDF expression and integral term, it is difficult to obtain a closed form solution from Equation 29. However, it can be evaluated through numerical approaches. Finally, substituting the analytical expressions for the individual NOMA users into Equation 28, we get the analytical expression for the overall sum capacity of the proposed BR NOMA-SWIPT system.

\section{G. TWR-OMA SWIPT System - For Benchmarking of Results}

In order to compare our proposed BR NOMA-SWIPT System, we investigate the performance of the TWR-OMA SWIPT system. Here, the terms TWR and BR are used interchangeably. Intuitively, comparing the performance of our proposed system with conventional BR OMA and NOMA techniques seems unreasonable as in conventional BR OMA and NOMA, only two user nodes can exchange information through a relay node. But, in our proposed system model, two NOMA user groups can exchange information through the EH based relay. Moreover, the impact of the EH factor also plays an important role in the overall performance of the system. Therefore, for a fair comparison of our results, we devise and investigate the performance of the TWR-OMA SWIPT system considering time division multiple access (TDMA) as a benchmark. For the TWR-OMA SWIPT system, we have considered the same system model as for the proposed BR NOMA-SWIPT system. Since we have considered TDMA for the TWR-OMA SWIPT system, the total communication process can be divided into eight-time slots. In the first time 
TABLE I

SIMULATION PARAMETERS

\begin{tabular}{|l|l|l|}
\hline Parameter & Symbol & Values \\
\hline Distance between R and $S_{1}$ & $d_{S_{1}}$ & $2 \mathrm{~m}, 0.25 \mathrm{~m}$ \\
Distance between R and $S_{2}$ & $d_{S_{2}}$ & $10 \mathrm{~m}, 0.50 \mathrm{~m}$ \\
Distance between R and $D_{1}$ & $d_{D_{1}}$ & $10 \mathrm{~m}, 0.50 \mathrm{~m}$ \\
Distance between R and $D_{2}$ & $d_{D_{2}}$ & $2 \mathrm{~m}, 0.25 \mathrm{~m}$ \\
Path Loss Factor & $\mathrm{v}$ & 2 \\
Transmit SNR & $\rho$ & $0-50 \mathrm{~dB}$ \\
Residual Interfering signal & $\xi$ & 0.03 \\
Energy Harvesting Efficiency & $\eta$ & 0.9 \\
$S_{1}$, and $S_{2}$ Data Rate & $R_{S_{1}}, R_{S_{2}}$ & $0.1 \mathrm{bps} / \mathrm{Hz}$ \\
$D_{1}$, and $D_{2}$ Data Rate & $R_{D_{1}}, R_{D_{2}}$ & $0.1 \mathrm{bps} / \mathrm{Hz}$ \\
Power Factor for NOMA & $a_{1}, b_{2}$ & 0.8 \\
Power Factor for NOMA & $a_{2}, b_{1}$ & 0.2 \\
Power Factor for NOMA & $a_{3}, b_{4}$ & 0.1 \\
Power Factor for NOMA & $a_{4}, b_{3}$ & 0.4 \\
\hline
\end{tabular}

slot, $S_{1}$ in group $\mathrm{S}$ sends its message to the EH based relay node R. R first harvests the energy from the signal of $S_{1}$ to recharge itself and for information decoding. In the next time slot, R sends the message of $S_{1}$ to the $D_{1}$ in group D. The same process is repeated for all other nodes in the remaining time slots. Finally, through this process, all the messages have been exchanged in the TWR-OMA SWIPT system. The sumthroughput of the TWR-OMA SWIPT system is given by:

$$
\begin{aligned}
T h r_{O M A}^{\text {sum }}= & \frac{1-\operatorname{Pr}\left(\min \left(\rho\left|h_{1}\right|^{2}, P_{e}\left|h_{1}\right|^{2}\left|g_{1}\right|^{2}\right)<\gamma\right)(1-\alpha) R_{S_{1}}}{8}+ \\
& \frac{1-\operatorname{Pr}\left(\min \left(\rho\left|g_{1}\right|^{2}, P_{e}\left|h_{1}\right|^{2}\left|g_{1}\right|^{2}\right)<\gamma\right)(1-\alpha) R_{D_{1}}}{8}+ \\
& \frac{1-\operatorname{Pr}\left(\min \left(\rho\left|h_{2}\right|^{2}, P_{e}\left|h_{2}\right|^{2}\left|g_{2}\right|^{2}\right)<\gamma\right)(1-\alpha) R_{S_{2}}}{8}+ \\
& \frac{1-\operatorname{Pr}\left(\min \left(\rho\left|g_{2}\right|^{2}, P_{e}\left|h_{2}\right|^{2}\left|g_{2}\right|^{2}\right)<\gamma\right)(1-\alpha) R_{D_{2}}}{8}
\end{aligned}
$$

where $P_{e}=\frac{8 \eta \alpha \rho}{(1-\alpha)}$.

Similarly, the ergodic sum capacity of the TWR-OMA SWIPT system is given by:

$$
\begin{aligned}
C_{O M A}^{\text {sum }}= & \frac{(1-\alpha)}{8} E\left[\log _{2}\left(1+\min \left(\rho\left|h_{1}\right|^{2}, P_{e}\left|h_{1}\right|^{2}\left|g_{1}\right|^{2}\right)\right]+\right. \\
& \frac{(1-\alpha)}{8} E\left[\log _{2}\left(1+\min \left(\rho\left|g_{1}\right|^{2}, P_{e}\left|h_{1}\right|^{2}\left|g_{1}\right|^{2}\right)\right]+\right. \\
& \frac{(1-\alpha)}{8} E\left[\log _{2}\left(1+\min \left(\rho\left|h_{2}\right|^{2}, P_{e}\left|h_{2}\right|^{2}\left|g_{2}\right|^{2}\right)\right]+\right. \\
& \frac{(1-\alpha)}{8} E\left[\log _{2}\left(1+\min \left(\rho\left|g_{2}\right|^{2}, P_{e}\left|h_{2}\right|^{2}\left|g_{2}\right|^{2}\right)\right]\right.
\end{aligned}
$$

\section{Numerical Results AND Discussions}

In this section, we present our Monte-Carlo simulation results to verify our analytical derivations of the BR NOMASWIPT system. We have used the simulation parameters listed in Table I unless otherwise stated. MATLAB is running the Monte-Carlo simulations by averaging over $10^{5}$ random realizations of Rayleigh block fading channels $h_{1}, h_{2}, g_{1}, g_{2}$

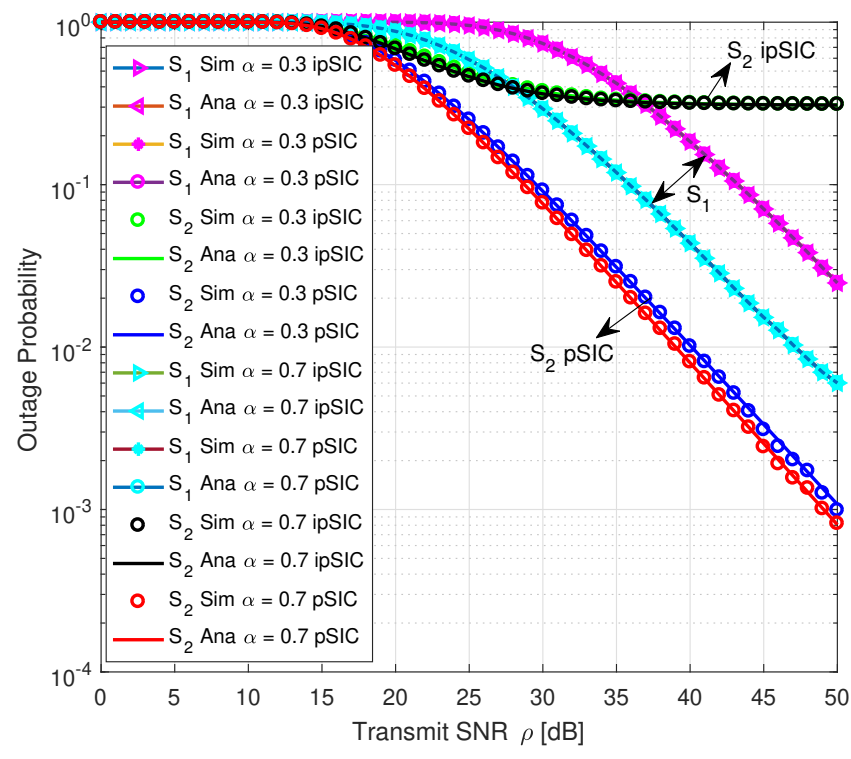

Fig. 4. Outage probability of the BR NOMA-SWIPT system

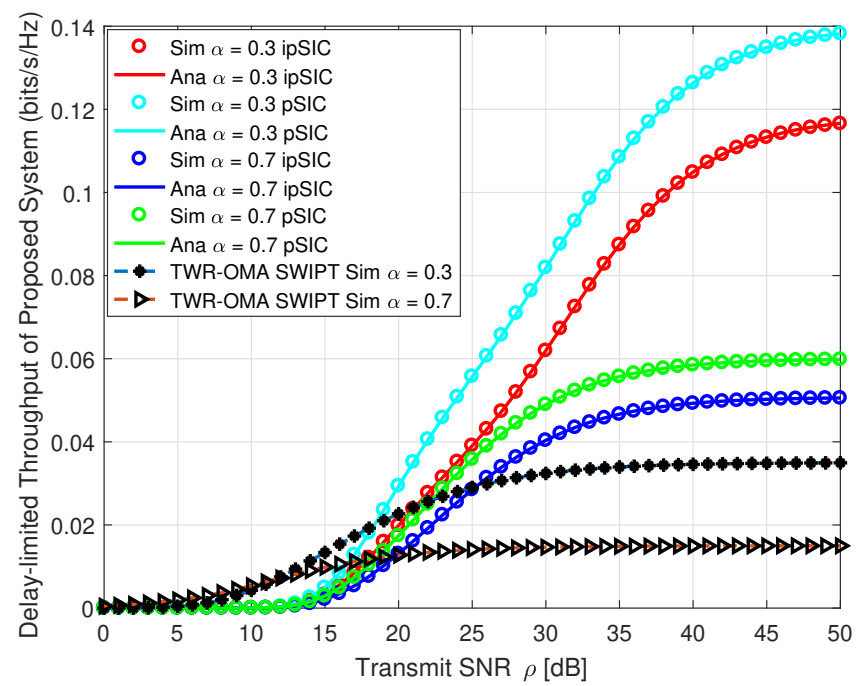

Fig. 5. Sum-throughput of the BR NOMA-SWIPT system

to get the simulation results.

In Fig. 4, we plot the outage probability of $S_{1}$ and $S_{2}$ with both pSIC and ipSIC. Since, we have assumed channel reciprocity, the outage probabilities of $D_{1}$ and $D_{2}$ are almost similar, and hence they are omitted for the sake of brevity. In Fig. 4, we observe on $S_{1}$, which is a near NOMA user sees hardly any effect of ipSIC. Hence pSIC and ipSIC results are almost the same for $S_{1}$. But, we can clearly see that ipSIC makes a difference in the outage probability for the far $S_{2}$ NOMA user. Due to the impact of residual inference, the outage probability of $S_{2}$ is higher, and we can see the significant difference in the outage probability for pSIC and ipSIC when the transmit SNR os higher than $15 \mathrm{~dB}$. This indicates that the ipSIC has a dominant effect on the outage probability of $S_{2}$. Since the relay node harvests the RF energy 


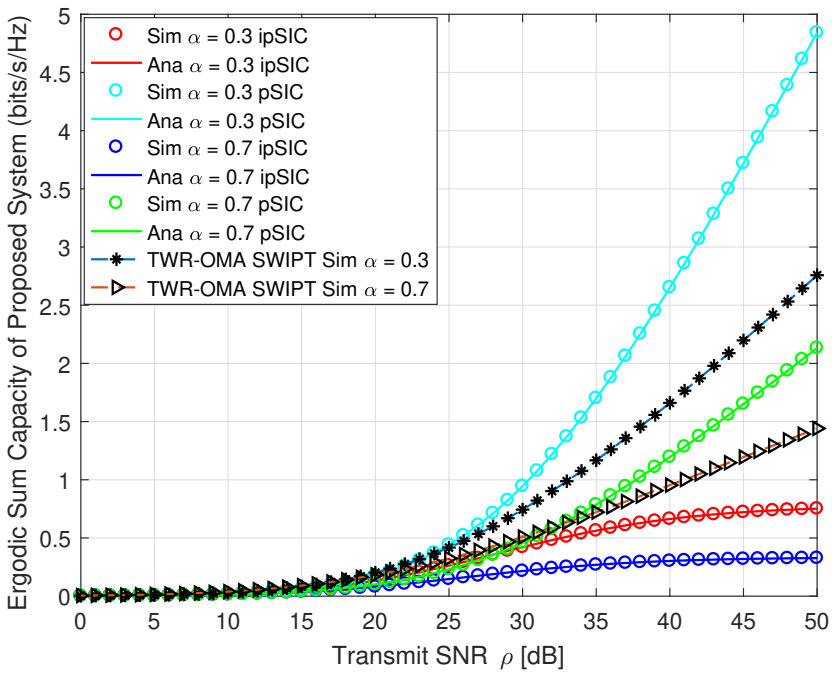

Fig. 6. Ergodic sum capacity of the BR NOMA-SWIPT system

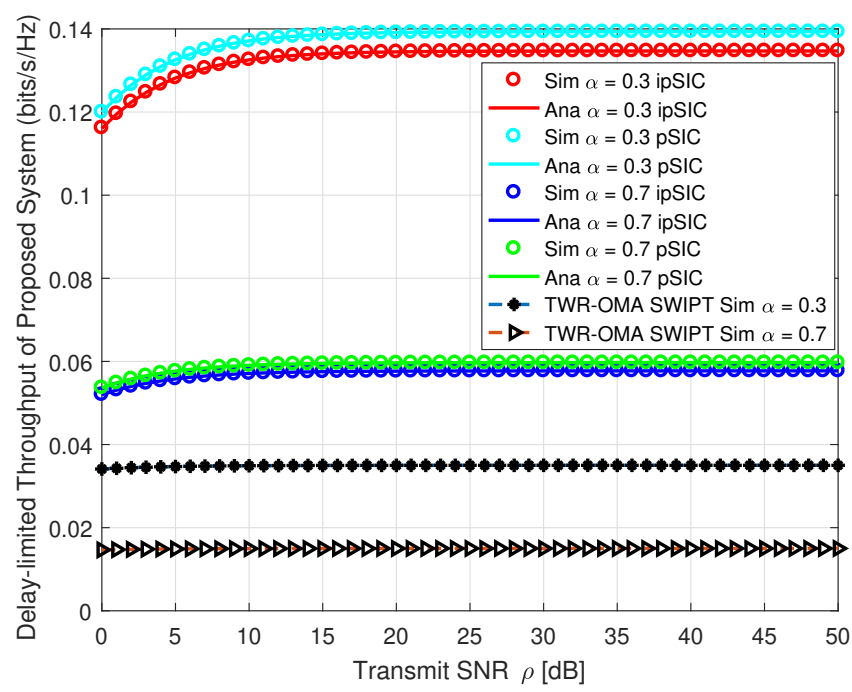

Fig. 7. Sum-throughput of the BR NOMA-SWIPT system with short distance$d_{S_{1}}=d_{D_{2}}=0.25 \mathrm{~m}$ and $d_{S_{2}}=d_{D_{1}}=0.50 \mathrm{~m}$

from the signals of the NOMA users in groups $\mathrm{S}$ and $\mathrm{D}$, the transmit SNR also plays an important role here. As we notice, the outage probabilities of $S_{1}$ and $S_{2}$ are almost 1 when the transmit SNR is less than $15 \mathrm{~dB}$ for both pSIC and ipSIC. The reason for this is that $\mathrm{R}$ is able to harvest more energy at higher transmit SNR, i.e. greater than $15 \mathrm{~dB}$, which tends to lower the outage probability. Also, the time switching factor $\alpha$ tends to lower the outage probability as we increase it from 0.3 to 0.7 . Moreover, we observe that the outage probability of $S_{2}$ with ipSIC is almost saturated at an SNR greater than $35 \mathrm{~dB}$, whereas the outage probability of $S_{1}$ decreases with an increasing transmit SNR. Apparently, we also observe that the outage probability curve matches exactly the simulation results which indicates that our derived analysis of the outage probability of the BR NOMA-SWIPT system is correct.

In Fig. 5 and Fig. 6, we plot the sum-throughput and ergodic

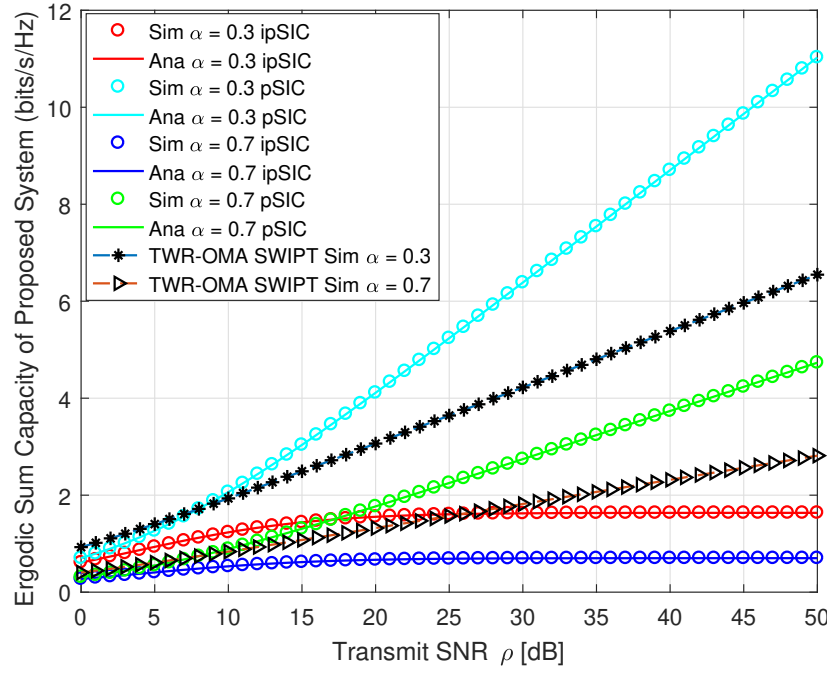

Fig. 8. Ergodic sum capacity of the BR NOMA-SWIPT system with short distance $-d_{S_{1}}=d_{D_{2}}=0.25 \mathrm{~m}$ and $d_{S_{2}}=d_{D_{1}}=0.50 \mathrm{~m}$

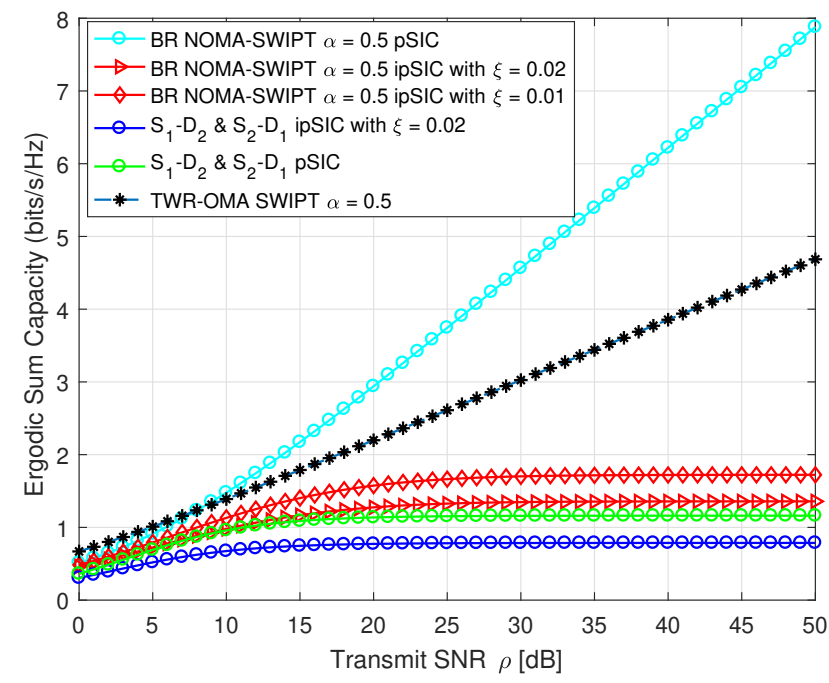

Fig. 9. Ergodic sum capacity comparison of the BR NOMA-SWIPT system with different user pairing

sum capacity of the proposed BR NOMA-SWIPT system. For a fair comparison or benchmarking, we also plotted the sum-throughput and ergodic sum capacity of the TWR-OMA SWIPT system. We see that the sum-throughput of the TWROMA SWIPT is higher than that of BR NOMA-SWIPT for a transmit SNR 0-15 dB after which it becomes saturated at a higher transmit SNR. We also observe the significant improvement of the sum-throughput of our BR NOMA-SWIPT after $20 \mathrm{~dB}$ transmit SNR. This indicates that for the BR NOMA-SWIPT to have a higher sum-throughput performance we need a transmit SNR greater than $20 \mathrm{~dB}$. This is expected, since ipSIC tends to lower the sum-throughput performance. Furthermore, Fig. 5 shows that an increase in $\alpha$ from 0.3 to 0.7 , reduces the sum-throughput of the proposed system especially when the transmit SNR is higher than $20 \mathrm{~dB}$. This is because 


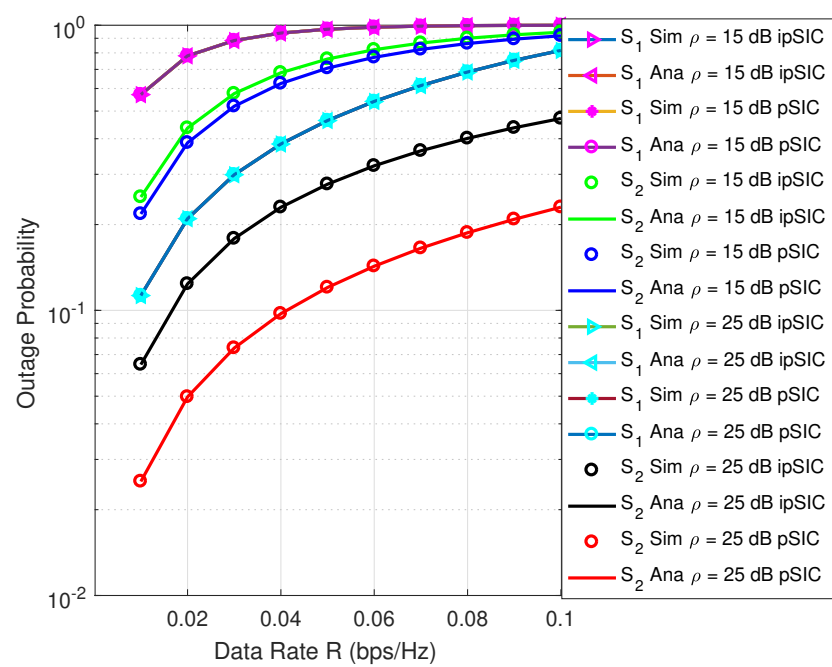

Fig. 10. Outage probability at different data rate of the BR NOMA-SWIPT system

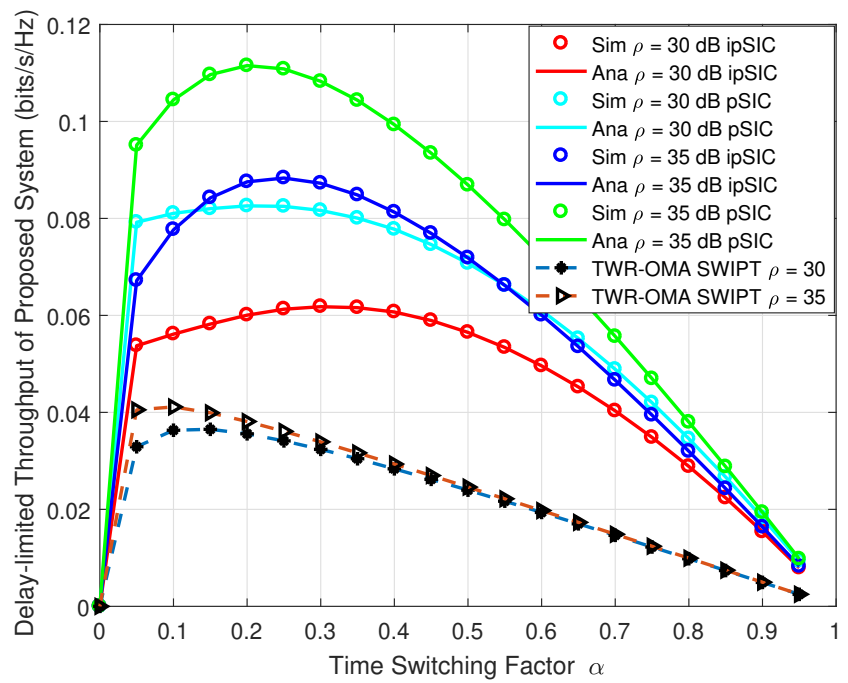

Fig. 11. Sum-throughput vs. $\alpha$ with different $\rho$ for the BR NOMA-SWIPT system

increasing the $\alpha$ value implies that less amount of time will be allocated to information decoding and data transmission at $\mathrm{R}$, which increases the outage in the system. Similarly, Fig. 6 shows a similar trend as in Fig. 5. An interesting thing to note in Fig. 6 is that the TWR-OMA SWIPT at $\alpha=0.3$ has a higher ergodic sum capacity performance than our proposed BR NOMA-SWIPT at a transmit SNR 0-25 dB. The ergodic capacity of the TWR-OMA SWIPT system at $\alpha=0.3$ even remains higher than that of the BR NOMA-SWIPT system with ipSIC. Also, we observe a noticeable performance improvement in the BR NOMA-SWIPT with pSIC at a higher transmit SNR than that of the TWR-OMA SWIPT and of the BR NOMA-SWIPT with ipSIC. This indicates that with the use of a better SIC technique, a significant improvement of the ergodic sum capacity can be achieved.

Next, we intend to verify the effect of the distance on our

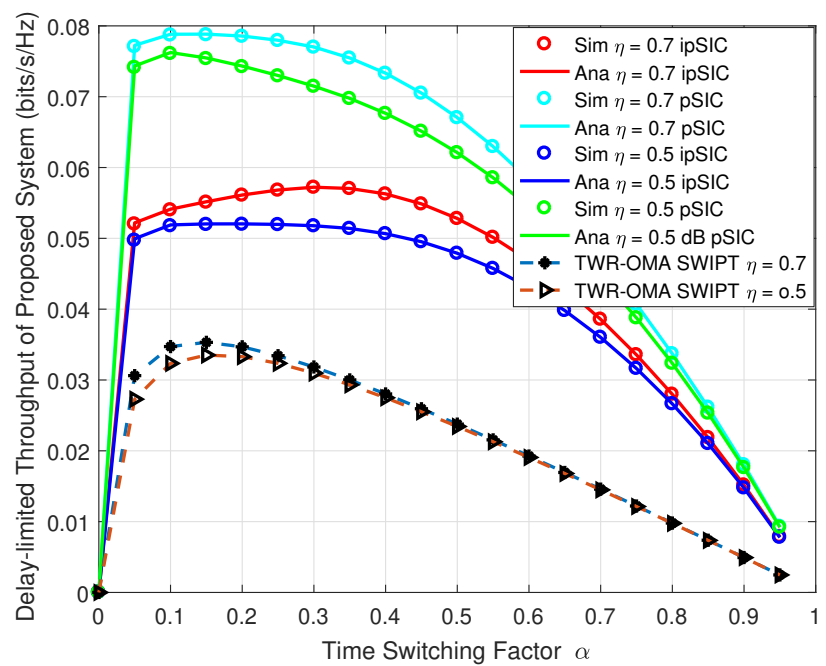

Fig. 12. Sum-throughput vs. $\alpha$ with different $\eta$ for the BR NOMA-SWIPT system

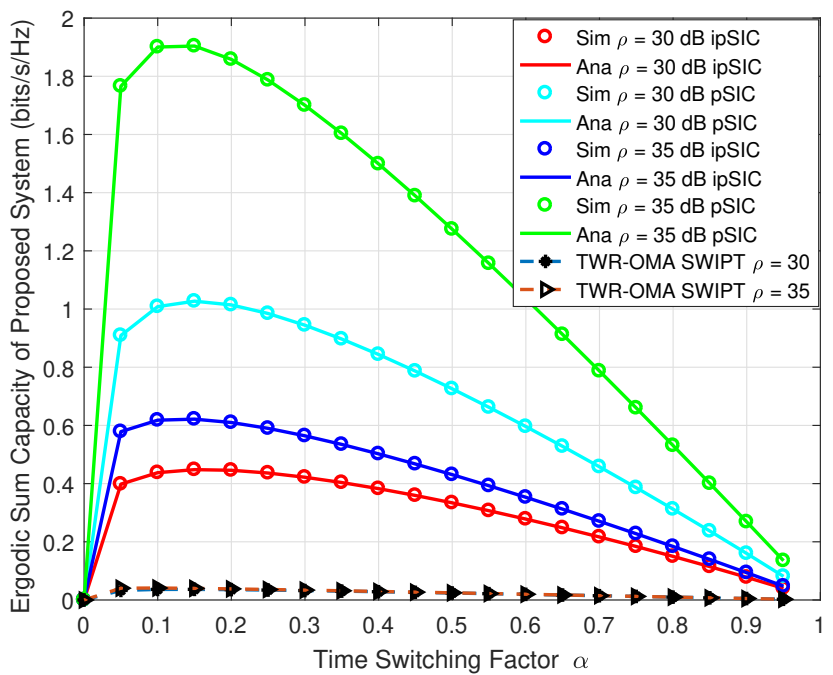

Fig. 13. Ergodic sum capacity vs. $\alpha$ with different $\rho$ for the BR NOMASWIPT system

proposed BR NOMA-SWIPT system. The distance of the relay node from the NOMA users plays an important role for RF $\mathrm{EH}$ and its impact can be clearly seen in the sum-throughput and ergodic sum capacity in Fig. 7 and Fig. 8 respectively. For plotting the results in Fig. 7 and Fig. 8, we set the distances between the NOMA group users and R to small values, such that $d_{S_{1}}=d_{D_{2}}=0.25 \mathrm{~m}$ and $d_{S_{2}}=d_{D_{1}}=0.50 \mathrm{~m}$. Unlike in Fig. 5 and Fig. 6, we observe that the sumthroughput and the ergodic sum capacity increase when the transmit SNR increases from $0 \mathrm{~dB}$ to $50 \mathrm{~dB}$. Moreover, the sum-throughput tends to increase upto $10 \mathrm{~dB}$ transmit SNR after which it becomes saturated for higher transmit SNR. However, the sum-throughput difference between pSIC and ipSIC cases in Fig. 7 is not much compared to the ergodic sum capacity difference between pSIC and ipSIC cases in Fig. 8. Also, the sum-throughput performance of the TWR- 
OMA SWIPT system is lower than the sum-throughput of BR NOMA-SWIPT at all transmit SNR values from 0-50 dB. The ergodic sum capacity with pSIC is shown to give a higher performance than the TWR-OMA SWIPT at all transmit SNR values in Fig. 8, which implies that better SIC technique will improve the ergodic sum capacity of the BR NOMA-SWIPT. Also, a significant difference between the sum-throughput and ergodic sum capacity can be observed when we decrease the $\alpha$ value from 0.3 to 0.7 . A lower value of $\alpha$ tends to improve the performance of the BR NOMA-SWIPT such that more time can be allocated to the information decoding and data transmission at $\mathrm{R}$.

In Fig. 9 shows the effect of user pairing on the performance of sum capacity maximization of the BR NOMA-SWIPT system. Therefore, we consider the user pairing case where a NOMA user in group $\mathrm{S}$ with good channel condition i.e., $S_{1}$ exchanges information with another NOMA user in group D with good channel condition of group D i.e., $D_{2}$ and a NOMA user in group $\mathrm{S}$ with poor channel condition i.e., $S_{2}$ exchanges information with the NOMA user in group D with poor channel condition i.e., $D_{1}$ with the help of the EH based relay R. We see that the ergodic sum capacity of such a system is greatly affected when such user pairing is done, and the performance is severely degraded compared to our proposed BR NOMA-SWIPT and TWR-OMA SWIPT. This is because SIC is performed on different NOMA users during the uplink and downlink, and hence a user will not gain the appropriate benefits of SIC and cooperative diversity [34]. For our proposed BR NOMA-SWIPT system, SIC is performed on the same user during the uplink and downlink so that the sum capacity is maximized. Also, in Fig. 9, we observe that as $\xi$ decreases from 0.02 to 0.01 , the ergodic sum capacity of BR NOMA-SWIPT is improved.

We have set $\alpha=0.5$ in Fig. 10. Since the outage probability depends on the data rate of the $S_{1}, D_{1}, S_{2}$ and $D_{2}$, it is important to study its effect on the system. In Fig. 10, we observe that the outage probability of $S_{1}$ and $S_{2}$ with pSIC and ipSIC increases with an increasing data rate. This implies that our proposed BR NOMA-SWIPT system is expected to work well for applications with low data rate requirements, such as short queries and requests in light-weight IoT applications. Since, in BR NOMA-SWIPT system, the relay node is harvesting the energy from the NOMA uplink signal, it can only support applications with low data rate requirements to support its own data transmission, as only a small amount of energy can be harvested from the uplink NOMA signals. Hence, choosing a small and appropriate data rate could improve the outage probability and the sum-throughput of the BR NOMA-SWIPT system. Fig. 10 also shows that the outage probability of the $S_{1}$ is higher than that of the $S_{2}$ with the increase in data rate. This is because, $S_{1}$ need to satisfy its own data rate requirement and that of $D_{1}$ and $D_{2}$, whereas $S_{2}$ only need to satisfy its own data rate requirement and that of $D_{2}$. As expected, increasing the transmit SNR lowers the outage probability of the $S_{1}$ and $S_{2}$ with both pSIC and ipSIC.

In Fig. 11 and Fig. 12, we plot the sum-throughput of the BR NOMA-SWIPT system and the TWR-OMA SWIPT system against the time switching factor $\alpha$. We observe that, with an increase in $\alpha$, the sum-throughput first increases, then reaches a maximum and finally decreases. This implies that the sumthroughput is a concave function that has a unique maxima on the interval $[0,1]$ at which the sum-throughput is maximized. Furthermore, we observe that pSIC tends to lower the $\alpha$ value compared to ipSIC. Moreover, the energy conversion efficiency $\eta$ also has an impact on the sum-throughput of the BR NOMA-SWIPT system. As we decrease the $\eta$ from 0.7 to 0.5 , the sum-throughput also decreases as seen in Fig. 12 . Similarly, in Fig. 13, we plot the ergodic sum capacity against the $\alpha$ factor. We observe a similar pattern as in Fig. 11. The ergodic sum capacity is also a concave function that has a unique maxima on the interval $[0,1]$ at which the ergodic sum capacity of the BR NOMA-SWIPT system is maximized. In general, we cannot have a high value of $\alpha$ as less time will be allocated to information decoding and data transmission. This will directly impact the sum-throughput and the ergodic sum capacity performance of the system. Therefore, finding an optimum $\alpha$ is important for maximization of the sumthroughput and the ergodic sum capacity. Optimum $\alpha$ can be easily found through iterative search algorithm, such as Golden section search method [40].

\section{CONClusion AND Future Works}

Aiming to increase the spectral efficiency and energy efficiency, we proposed a BR NOMA-SWIPT system and investigated its performance for wireless IoT relay networks. In BR NOMA-SWIPT, NOMA users in one group can exchange information with NOMA users in another group with the help of an energy-constrained relay node (R). The outage probability, throughput, and ergodic capacity of each user and the sum-throughput and sum capacity of the BR NOMASWIPT system were analytically derived under both pSIC and ipSIC scenarios. The proposed BR NOMA-SWIPT system showed an enhancement in the sum-throughput and sum capacity as compared to TWR-OMA SWIPT system. The effect of user pairing was also studied and it showed the capacity enhancement of the BR NOMA-SWIPT system. Our results also revealed that the ipSIC has a significant effect on the performance of the BR NOMA-SWIPT system as compared to pSIC scenario. This demonstrated the need for pSIC techniques for capacity and throughput enhancements of the system. The effect of the EH parameters, such as the time switching factor and the energy conversion efficiency, was also studied to get an insight into the sum-throughput and sum capacity maximization of the BR NOMA-SWIPT system. Finally, it is evident that our proposed BR NOMA-SWIPT system is feasible for ubiquitous IoT networks with low data rate requirements for self-sustainable and energy-efficient data transmissions.

Since this is one of the initial attempts to address and use BR NOMA with SWIPT that supports low data rate requirement of IoT applications, in future we plan to model system that will support also high data rate requirements of NOMA users according to their quality of service (QoS) requirements. Moreover, we plan to extend our model to study secrecy capacity for secure communication in IoT relay networks. 
Nevertheless, evaluating the performance of our system with other multipath fading channel model is the interest of our future work.

\section{APPENDIX A} PROOF OF THEOREM 1

$P_{S_{1}}^{\text {Out }}=1-\operatorname{Pr}\left(\gamma_{R \rightarrow S_{1}}>\gamma_{S_{1}}^{T}, \gamma_{D_{1} \rightarrow S_{1}}>\gamma_{D_{1}}^{T}, \gamma_{D_{2} \rightarrow S_{1}}>\gamma_{D_{2}}^{T}\right)$ Let $\gamma_{S_{1}}^{T}=\gamma_{D_{1}}^{T}=\gamma_{D_{2}}^{T}=\gamma$ $=1-\operatorname{Pr}\left(\frac{\rho a_{1}\left|h_{1}\right|^{2}}{\rho a_{2}\left|h_{2}\right|^{2}+1}>\gamma, \frac{a_{3} P_{R}\left|g_{1}\right|^{2}}{a_{4} P_{R}\left|g_{1}\right|^{2}+1}>\gamma, \frac{a_{3} P_{R}\left|g_{2}\right|^{2}}{a_{4} P_{R}\left|g_{2}\right|^{2}+1}>\gamma\right)$ Let, $a_{1}\left|h_{1}\right|^{2}=X_{1}, a_{2}\left|h_{2}\right|^{2}=X_{2}, b_{1}\left|g_{1}\right|^{2}=Y_{1}, b_{2}\left|g_{2}\right|^{2}=Y_{2}$ $P_{R}=c\left(X_{1}+X_{2}+Y_{1}+Y_{2}\right)$, where $\mathrm{c}=\frac{2 \eta \alpha P_{T}}{(1-\alpha)}$ $=1-\operatorname{Pr}\left(\frac{\rho X_{1}}{\rho X_{2}+1}>\gamma, \frac{a_{3} c Z_{1}}{a_{4} c Z_{1}+b_{1}}>\gamma, \frac{a_{3} c Z_{2}}{a_{4} c Z_{2}+b_{2}}>\gamma\right)$ where $Z_{1}=Y_{1}\left(X_{1}+X_{2}+Y_{1}+Y_{2}\right)$ and $Z_{2}=Y_{2}\left(X_{1}+X_{2}+Y_{1}+Y_{2}\right)$

Since, the fraction $\frac{a_{3} c Z_{1}}{a_{4} c Z_{1}+b_{1}}$ and $\frac{a_{3} c Z_{2}}{a_{4} c Z_{2}+b_{2}}$ never exceeds $\frac{a_{3}}{a_{4}}$, we have, $P_{S_{1}}^{\text {Out }}=0$ for $\gamma \geq \frac{a_{3}}{a_{4}}$

For $0 \leq \gamma \leq \frac{a_{3}}{a_{4}}$, we have,

$P_{S_{1}}^{\text {Out }}=1-\operatorname{Pr}\left(X_{1}>\gamma X_{2}+\frac{\gamma}{\rho}, Y_{1}\left(X_{1}+X_{2}+Y_{1}+Y_{2}\right)>\right.$ $\left.\frac{b_{1} \gamma}{c\left(a_{3}-a_{4} \gamma\right)}, Y_{2}\left(X_{1}+X_{2}+Y_{1}+Y_{2}\right)>\frac{b_{2} \gamma}{c\left(a_{3}-a_{4} \gamma\right)}\right)$

Let, $\frac{\gamma}{\rho}=t, \frac{b_{1} \gamma}{c\left(a_{3}-a_{4} \gamma\right)}=z_{1}, \frac{b_{2} \gamma}{c\left(a_{3}-a_{4} \gamma\right)}=z_{2}$

$Q_{T}\left(\gamma, t, z_{1}, z_{2}\right)=\operatorname{Pr}\left(X_{1}>\gamma X_{2}+t, Y_{1}\left(X_{1}+X_{2}+Y_{1}+Y_{2}\right)>z_{1}\right.$, $\left.Y_{2}\left(X_{1}+X_{2}+Y_{1}+Y_{2}\right)>z_{2}\right)$

Now, conditioning $Q_{T}\left(\gamma, t, z_{1}, z_{2}\right)$ on $Y_{1}$ and $Y_{2}$, we get, $Q_{T}\left(\gamma, t, z_{1}, z_{2}\right)=\int_{y_{1}=0}^{\infty} \int_{y_{2}=0}^{\infty} \operatorname{Pr}\left(X_{1}>\gamma X_{2}+t, X_{1}+X_{2}>\right.$ $\left.\frac{z_{1}}{y_{1}}-y_{1}-y_{2}, X_{1}+X_{2}>\frac{z_{2}}{y_{2}}-y_{1}-y_{2}\right) f_{Y_{2}}\left(y_{2}\right) d y_{2} f_{Y_{1}}\left(y_{1}\right) d y_{1}$ $Q_{T}\left(\gamma, t, z_{1}, z_{2}\right)=\int_{y_{1}=0}^{\infty} \int_{y_{2}=0}^{\infty}$ $\operatorname{Pr}\left(X_{1}>\gamma X_{2}+t, X_{1}+X_{2}>\max \left[\frac{z_{1}}{y_{1}}, \frac{z_{2}}{y_{2}}\right]-y_{1}-y_{2}\right)$ $G(\gamma, t, s)$

$f_{Y_{2}}\left(y_{2}\right) d y_{2} f_{Y_{1}}\left(y_{1}\right) d y_{1}$
$Q_{T}\left(\gamma, t, z_{1}, z_{2}\right)=\int_{y_{1}=0}^{\infty} \int_{y_{2}=0}^{\infty} G\left(\gamma, t, \max \left[\frac{z_{1}}{y_{1}}, \frac{z_{2}}{y_{2}}\right]-y_{1}-y_{2}\right)$

$f_{Y_{2}}\left(y_{2}\right) d y_{2} f_{Y_{1}}\left(y_{1}\right) d y_{1}$

$$
\begin{aligned}
& =\underbrace{\int_{y_{1}=0}^{\infty} \int_{y_{2}=0}^{y_{1} \frac{z_{2}}{z_{1}}} G\left(\gamma, t, \frac{z_{2}}{y_{2}}-y_{1}-y_{2}\right) f_{Y_{2}}\left(y_{2}\right) d y_{2} f_{Y_{1}}\left(y_{1}\right) d y_{1}}_{Q_{1}\left(\gamma, t, z_{1}, z_{2}\right)}+ \\
& \underbrace{\int_{y_{1}=0}^{\infty} \int_{y_{2}=y_{1} \frac{z_{2}}{z_{1}}}^{\infty} G\left(\gamma, t, \frac{z_{1}}{y_{1}}-y_{1}-y_{2}\right) f_{Y_{2}}\left(y_{2}\right) d y_{2} f_{Y_{1}}\left(y_{1}\right) d y_{1}}_{Q_{2}\left(\gamma, t, z_{1}, z_{2}\right)} \\
& Q_{T}\left(\gamma, t, z_{1}, z_{2}\right)=Q_{1}\left(\gamma, t, z_{1}, z_{2}\right)+Q_{2}\left(\gamma, t, z_{1}, z_{2}\right)
\end{aligned}
$$

Hence, we have,

$Q_{1}\left(\gamma, t, z_{1}, z_{2}\right)=\int_{y_{2}=0}^{\infty} H_{1}\left(\gamma, t, y_{2} \frac{z_{1}}{z_{2}}, \frac{z_{2}}{y_{2}}-y_{2}\right) f_{Y_{2}}\left(y_{2}\right) d y_{2}$ with $H_{1}(\gamma, t, v, x)=\int_{y_{1}=v}^{\infty} G\left(\gamma, t, x-y_{1}\right) f_{Y_{1}}\left(y_{1}\right) d y_{1}$

$Q_{2}\left(\gamma, t, z_{1}, z_{2}\right)=\int_{y_{1}=0}^{\infty} H_{2}\left(\gamma, t, y_{1} \frac{z_{2}}{z_{1}}, \frac{z_{1}}{y_{1}}-y_{1}\right) f_{Y_{1}}\left(y_{1}\right) d y_{1}$ with $H_{2}(\gamma, t, v, x)=\int_{y_{2}=v}^{\infty} G\left(\gamma, t, x-y_{2}\right) f_{Y_{2}}\left(y_{2}\right) d y_{2}$

Let us first evaluate,

$E(\gamma, t)=\operatorname{Pr}\left(X_{1}>\gamma X_{2}+t\right)$

Conditioning $E(\gamma, t)$ on $X_{2}$, we get,

$E(\gamma, t)=\int_{x_{2}=0}^{\infty} F_{X_{1}}\left(\gamma x_{2}+t\right) f_{X_{2}}\left(x_{2}\right) d x_{2}=\frac{\lambda_{h_{2}} e^{-\lambda_{h_{1}} t}}{\lambda_{h_{2}}+\gamma \lambda_{h_{1}}}$

Also, we have,

$G(\gamma, t, s)=\operatorname{Pr}\left(X_{1}>\gamma X_{2}+t, X_{1}+X_{2}>s\right)$

We can easily see that, for $s<t$,

$G(\gamma, t, s)=G^{-}(\gamma, t, s)=E(\gamma, t)=\frac{\lambda_{h_{2}} e^{-\lambda_{h_{1}} t}}{\lambda_{h_{2}}+\gamma \lambda_{h_{1}}}$

For $s>t$, we have,

$G(\gamma, t, s)=G^{+}(\gamma, t, s)=\int_{x_{2}=0}^{\frac{s-t}{1+\gamma}} F_{X_{1}}\left(s-x_{2}\right) f_{X_{2}}\left(x_{2}\right) d x_{2}+$ $\int_{x_{2}=\frac{s-t}{1+\gamma}}^{\infty} F_{X_{1}}\left(\gamma x_{2}+t\right) f_{X_{2}}\left(x_{2}\right) d x_{2}$

After some algebraic calculation, we can find that $G^{+}(\gamma, t, s)=\frac{\lambda_{h_{2}}}{\lambda_{h_{1}}-\lambda_{h_{2}}}\left(-e^{-\lambda_{h_{1}} s}+\frac{\lambda_{h_{1}}}{\eta_{1}} e^{-\left(\lambda_{h_{1}}-\lambda_{h_{2}}\right) \delta} e^{-\eta_{1} s}\right)$ where, $\eta_{1}=\frac{\lambda_{h_{2}}+\gamma \lambda_{h_{1}}}{1+\gamma}$ and, $\delta=\frac{t}{1+\gamma}$

Now, special attention must be put on the integrals of $Q_{1}$ and $Q_{2}$ functions, since, we have two expressions for $G(\gamma, t, s)$ i.e., whether $s<t$ or $s>t$. If, $x-v<t$ then $G^{-}\left(\gamma, t, x-y_{1}\right)=$ $E(\gamma, t)$ and take, $H_{1}(\gamma, t, v, x)=H_{1}^{-}(\gamma, t, v, x)=E(\gamma, t) F_{Y_{1}}(v)$. $H_{1}^{-}(\gamma, t, v, x)=\frac{\lambda_{h_{2}}}{\lambda_{h_{2}}+\gamma \lambda_{h_{1}}} e^{-\lambda_{h_{1}} t-\lambda_{g_{1}} v}$

If $x-v>t$ then the $H_{1}$ function integral splits into two parts: $H_{1}(\gamma, t, v, x)=H_{1}^{+}(\gamma, t, v, x)=\int_{y_{1}=v}^{x-t} G^{+}\left(\gamma, t, x-y_{1}\right) f_{Y_{1}}\left(y_{1}\right) d y_{1}$ 


$$
\begin{aligned}
& +\int_{y_{1}=x-t}^{\infty} G^{-}\left(\gamma, t, x-y_{1}\right) f_{Y_{1}}\left(y_{1}\right) d y_{1} \\
& H_{1}^{+}(\gamma, t, v, x)=\int_{y_{1}=v}^{x-t} G^{+}\left(\gamma, t, x-y_{1}\right) f_{Y_{1}}\left(y_{1}\right) d y_{1}+ \\
& E(\gamma, t) F_{Y_{1}}(x-t) \\
& H_{1}^{+}(\gamma, t, v, x)=\int_{y_{1}=v}^{x-t} \frac{\lambda_{h_{2}}}{\lambda_{h_{1}}-\lambda_{h_{2}}}\left(-e^{-\lambda_{h_{1}}\left(x-y_{2}\right)}+\right. \\
& \left.\frac{\lambda_{h_{1}}}{\eta_{1}} e^{-\left(\lambda_{h_{1}}-\lambda_{h_{2}}\right) \delta} e^{-\eta_{1}\left(x-y_{1}\right)}\right) \lambda_{g_{1}} e^{-\lambda_{g_{1}} y_{1}} d y_{1}+ \\
& \frac{\lambda_{h_{2}}}{\lambda_{h_{2}}+\gamma \lambda_{h_{1}}} e^{-\lambda_{h_{1}} t} e^{-\lambda_{g_{1}}(x-t)}
\end{aligned}
$$

Performing the integration and after some algebraic calculation, we can find that,

$H_{1}^{+}(\gamma, t, v, x)=\frac{\lambda_{h_{2}} \lambda_{g_{1}}}{\left(\lambda_{h_{1}}-\lambda_{h_{2}}\right)\left(\lambda_{g_{1}}-\lambda_{h_{1}}\right)} e^{-\lambda_{h_{1}} x-\left(\lambda_{g_{1}}-\lambda_{h_{1}}\right) v_{-}}$

$\frac{\lambda_{h_{1}} \lambda_{h_{2}} \lambda_{g_{1}}}{\eta_{1}\left(\lambda_{h_{1}}-\lambda_{h_{2}}\right)\left(\eta_{1}-\lambda_{g_{1}}\right)} e^{-\left(\lambda_{h_{1}}-\lambda_{h_{2}}\right) \delta-\eta_{1} x-\left(\lambda_{g_{1}}-\eta_{1}\right) v_{+}}$

$\frac{\lambda_{h_{1}} \lambda_{h_{2}}}{(1+\gamma)\left(\eta_{1}-\lambda_{g_{1}}\right)\left(\lambda_{h_{1}}-\lambda_{g_{1}}\right)} e^{-\left(\lambda_{h_{1}}-\lambda_{g_{1}}\right) t-\lambda_{g_{1}} x}$

Now, also for $Q_{1}$ function, we have to distinguish between the two expressions for $H_{1}(\gamma, t, v, x)$ when $x-v<t$ or $x-v>t$.

The limit occurs when $\frac{z_{2}}{y_{2}}-y_{2}-y_{2} \frac{z_{1}}{z_{2}}=t \rightarrow y_{2}=y_{2}^{g}=$ $\frac{z_{2}}{z_{1}+z_{2}}\left(\sqrt{\left(z_{1}+z_{2}\right)+\frac{t^{2}}{4}}-\frac{t}{2}\right)$

Hence, when $y_{2}<y_{2}^{g}$ we have, $H_{1}=H_{1}^{+}$and for $y_{2}>y_{2}^{g}$, we have $H_{1}=H_{1}^{-}$finally giving,

$$
\begin{aligned}
& Q_{1}\left(\gamma, t, z_{1}, z_{2}\right)=\int_{y_{2}=0}^{y_{2}^{g}} H_{1}^{+}\left(\gamma, t, y_{2} \frac{z_{1}}{z_{2}}, \frac{z_{2}}{y_{2}}-y_{2}\right) f_{Y_{2}}\left(y_{2}\right) d y_{2}+ \\
& E(\gamma, t) \int_{y_{2}=y_{2}^{g}}^{\infty} F_{Y_{1}}\left(y_{2} \frac{z_{1}}{z_{2}}\right) f_{Y_{2}}\left(y_{2}\right) d y_{2}
\end{aligned}
$$

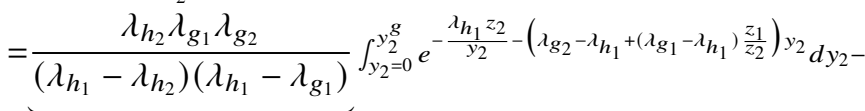

$$
\begin{aligned}
& \underbrace{}_{A} \\
& \underbrace{\frac{\lambda_{h_{1}} \lambda_{h_{2}} \lambda_{g_{1}} \lambda_{g_{2}} e^{-\left(\lambda_{h_{1}}-\lambda_{h_{2}}\right) \delta}}{\eta_{1}\left(\lambda_{h_{1}}-\lambda_{h_{2}}\right)\left(\eta_{1}-\lambda_{g_{1}}\right)}}_{B_{1}} \int_{y_{2}=0}^{y_{2}^{g}} e^{-\frac{\eta_{1} z_{2}}{y_{2}}-\left(\lambda_{g_{2}-\eta_{1}+\left(\lambda g_{1}-\eta_{1}\right)} \frac{z_{1}}{z_{2}}\right) y_{2}} d y_{2}+ \\
& \underbrace{\frac{\lambda_{h_{1}} \lambda_{h_{2}} \lambda_{g_{2}} e^{-\left(\lambda_{h_{1}}-\lambda_{g_{1}}\right) t}}{(1+\gamma)\left(\eta_{1}-\lambda_{g_{1}}\right)\left(\lambda_{h_{1}}-\lambda_{g_{1}}\right)}}_{C_{1}} \int_{y_{2}=0}^{y_{2}^{g}} e^{-\frac{\lambda_{g_{1} z_{2}}}{y_{2}}-\left(\lambda_{g_{2}}-\lambda_{g_{1}}\right) y_{2}} d y_{2}+ \\
& \underbrace{\frac{\lambda_{h_{2}} \lambda_{g_{2}} e^{-\lambda_{h_{1}} t-\left(\lambda_{g_{1}} \frac{z_{1}}{z_{2}}+\lambda_{g_{2}}\right) y_{2}^{g}}}{\left(\lambda_{g_{2}}+\gamma \lambda_{h_{1}}\right)\left(\lambda_{g_{1}} \frac{z_{1}}{z_{2}}+\lambda_{g_{2}}\right)}}_{D_{1}}
\end{aligned}
$$

The integral in above is in the form $\int_{x=0}^{a} e^{-\frac{c}{x}-b x} d x$ which can be solved in closed form [40] as: $\sum_{n=0}^{\infty} \frac{(-1)^{n}}{n !} b^{n} a^{n+1} E_{n+2}\left(\frac{c}{a}\right)$ where $E_{n+2}(a)=\int_{y=1}^{\infty} y^{-n-2} e^{-a y} d y$ is exponential integral of order $\mathrm{n}+2$.
$Q_{1}\left(\gamma, t, z_{1}, z_{2}\right)=A_{1} \sum_{n=0}^{\infty} \frac{(-1)^{n}}{n !}\left(\lambda_{g_{2}}-\lambda_{h_{1}}+\left(\lambda_{g_{1}}-\lambda_{h_{1}}\right) \frac{z_{1}}{z_{2}}\right)^{n}\left(y_{2}^{g}\right)^{n+1} E_{n+2}\left(\frac{\lambda_{h_{1}} z_{2}}{y_{2}^{g}}\right)-$

$B_{1} \sum_{n=0}^{\infty} \frac{(-1)^{n}}{n !}\left(\lambda_{g_{2}}-\eta_{1}+\left(\lambda_{g_{1}}-\eta_{1}\right) \frac{z_{1}}{z_{2}}\right)^{n}\left(y_{2}^{g}\right)^{n+1} E_{n+2}\left(\frac{\eta_{1} z_{2}}{y_{2}^{g}}\right)+$

$C_{1} \sum_{n=0}^{\infty} \frac{(-1)^{n}}{n !}\left(\lambda_{g_{2}}-\lambda_{g_{1}}\right)^{n}\left(y_{2}^{g}\right)^{n+1} E_{n+2}\left(\frac{\lambda_{g_{1}} z_{2}}{y_{2}^{g}}\right)+D_{1}$

Similarly, $Q_{2}\left(\gamma, t, z_{1}, z_{2}\right)$, can be simplified as:

$Q_{2}\left(\gamma, t, z_{1}, z_{2}\right)=A_{2} \sum_{n=0}^{\infty} \frac{(-1)^{n}}{n !}\left(\lambda_{g_{1}}-\lambda_{h_{1}}+\left(\lambda_{g_{2}}-\lambda_{h_{1}}\right) \frac{z_{2}}{z_{1}}\right)^{n}\left(y_{1}^{g}\right)^{n+1} E_{n+2}\left(\frac{\lambda_{h_{1}} z_{1}}{y_{1}^{g}}\right)-$

$B_{2} \sum_{n=0}^{\infty} \frac{(-1)^{n}}{n !}\left(\lambda_{g_{1}}-\eta_{1}+\left(\lambda_{g_{2}}-\eta_{1}\right) \frac{z_{2}}{z_{1}}\right)^{n}\left(y_{1}^{g}\right)^{n+1} E_{n+2}\left(\frac{\eta_{1} z_{1}}{y_{1}^{g}}\right)+$

$C_{2} \sum_{n=0}^{\infty} \frac{(-1)^{n}}{n !}\left(\lambda_{g_{1}}-\lambda_{g_{2}}\right)^{n}\left(y_{1}^{g}\right)^{n+1} E_{n+2}\left(\frac{\lambda_{g_{2}} z_{1}}{y_{1}^{g}}\right)+D_{2}$

where, $A_{2}=\frac{\lambda_{h_{2}} \lambda_{g_{1}} \lambda_{g_{2}}}{\left(\lambda_{h_{1}}-\lambda_{h_{2}}\right)\left(\lambda_{h_{1}}-\lambda_{g_{2}}\right)}, B_{2}=\frac{\lambda_{h_{1}} \lambda_{h_{2}} \lambda_{g_{1}} \lambda_{g_{2}} e^{-\left(\lambda_{h_{1}}-\lambda_{h_{2}}\right) \delta}}{\eta_{1}\left(\lambda_{h_{1}}-\lambda_{h_{2}}\right)\left(\eta_{1}-\lambda_{g_{2}}\right)}$,

$C_{2}=\frac{\lambda_{h_{1}} \lambda_{h_{2}} \lambda_{g_{2}} e^{-\left(\lambda_{h_{1}}-\lambda_{g_{2}}\right) t}}{(1+\gamma)\left(\eta_{1}-\lambda_{g_{2}}\right)\left(\lambda_{h_{1}}-\lambda_{g_{2}}\right)}, D_{2}=\frac{\lambda_{h_{2}} \lambda_{g_{1}} e^{-\lambda_{h_{1}} t-\left(\lambda_{g_{2}} \frac{z_{1}}{z_{2}}+\lambda_{g_{1}}\right) y_{1}^{g}}}{\left(\lambda_{g_{2}}+\gamma \lambda_{h_{1}}\right)\left(\lambda_{g_{2}} \frac{z_{2}}{z_{1}}+\lambda_{g_{1}}\right)}$

Therefore,

$P_{S_{1}}^{\text {Out }}=1-\left(Q_{1}\left(\gamma, t, z_{1}, z_{2}\right)+Q_{2}\left(\gamma, t, z_{1}, z_{2}\right)\right)$

Substituting $Q_{1}\left(\gamma, t, z_{1}, z_{2}\right)$ and $Q_{2}\left(\gamma, t, z_{1}, z_{2}\right)$ above, we get the final expressions as in Equation 18.

This completes the proof of Theorem 1.

\section{APPENDIX B}

PROOF OF THEOREM 2

$P_{S_{2}}^{\text {Out }}=1-\operatorname{Pr}\left(\gamma_{R \rightarrow S_{2}}>\gamma_{t h S_{2}}, \gamma_{D_{2} \rightarrow S_{2}}>\gamma_{t h D_{2}}\right)$

Let $\gamma_{t h S_{2}}=\gamma_{t h D_{2}}=\gamma$

$$
=1-\operatorname{Pr}\left(\frac{\rho a_{2}\left|h_{2}\right|^{2}}{\rho a_{1} \xi\left|h_{1}\right|^{2}+1}>\gamma, \frac{a_{4} P_{R}\left|g_{2}\right|^{2}}{a_{3} P_{R} \xi\left|g_{2}\right|^{2}+1}>\gamma\right)
$$

Let, $a_{1}\left|h_{1}\right|^{2}=X_{1}, a_{2}\left|h_{2}\right|^{2}=X_{2}, b_{1}\left|g_{1}\right|^{2}=Y_{1}, b_{2}\left|g_{2}\right|^{2}=Y_{2}$

$P_{R}=c\left(X_{1}+X_{2}+Y_{1}+Y_{2}\right)$, where $\mathrm{c}=\frac{2 \eta \alpha P_{T}}{(1-\alpha)}$

$=1-\operatorname{Pr}\left(\frac{\rho X_{2}}{\rho \xi X_{1}+1}>\gamma, \frac{\frac{a_{4}}{b_{2}} c b_{2} Y_{2}\left(X_{1}+X_{2}+Y_{1}+Y_{2}\right)}{\frac{a_{3}}{b_{2}} \xi c b_{2} Y_{2}\left(X_{1}+X_{2}+Y_{1}+Y_{2}\right)+1}>\gamma\right)$

Let $d_{1}=\frac{a_{4}}{b_{2}} c, d_{2}=\frac{a_{3} \xi c}{b_{2}}, Z=Y_{2}\left(X_{1}+X_{2}+Y_{1}+Y_{2}\right)$

$=1-\operatorname{Pr}\left(\frac{\rho X_{2}}{\rho \xi X_{1}+1}>\gamma, \frac{d_{1} Z}{d_{2} Z+1}>\gamma\right)$

$=1-\operatorname{Pr}\left(X_{2}>\xi \gamma X_{1}+\frac{\gamma}{\rho}, Z>\frac{\gamma}{d_{1}-\gamma d_{2}}\right)$

Let, $k=\frac{\gamma}{\rho}, t=\frac{\gamma}{d_{1}-\gamma d_{2}}$

$=1-\underbrace{\operatorname{Pr}\left(X_{2}>\xi \gamma X_{1}+k, Y_{2}\left(X_{1}+X_{2}+Y_{1}+Y_{2}\right)>t\right)}$

$Q(\xi, \gamma, k, t)$

Now, 
Conditioning $Q(\xi, \gamma, k, t)$ on $Y_{2}$, we get,

$$
\begin{aligned}
& =\int_{y_{2}=0}^{\infty} \operatorname{Pr}\left(X_{2}>\xi \gamma X_{1}+k, y_{2}\left(X_{1}+X_{2}+Y_{1}+y_{2}\right)>t\right) f_{Y_{2}}\left(y_{2}\right) d y_{2} \\
& =\int_{y_{2}=0}^{\infty} \operatorname{Pr}(X_{2}>\xi \gamma X_{1}+k,\left(X_{1}+X_{2}+Y_{1}\right)>\underbrace{\frac{t}{y_{2}}-y_{2}}_{u}) f_{Y_{2}}\left(y_{2}\right) d y_{2}
\end{aligned}
$$

Let us first evaluate $F(\xi, \gamma, k)$

$F(\xi, \gamma, k)=\operatorname{Pr}\left(X_{2}>\xi \gamma X_{1}+k\right)$

Conditioning $F(\xi, \gamma, k)$ on $X_{1}$, we get,

$F(\xi, \gamma, k)=\int_{x_{1}=0}^{\infty} \operatorname{Pr}\left(X_{2}>\xi \gamma x_{1}+k\right) f_{X_{1}}\left(x_{1}\right) d x_{1}$

$F(\xi, \gamma, k)=\frac{\lambda_{h_{1}} e^{-k \lambda_{h_{2}}}}{\xi \gamma \lambda_{h_{2}}+\lambda_{h_{1}}}$

Now,

$H(\xi, \gamma, k, u)=\operatorname{Pr}\left(X_{2}>\xi \gamma X_{1}+k, X_{1}+X_{2}+Y_{1}>u\right)$

Conditioning $H(\xi, \gamma, k, u)$ on $Y_{1}$, we get,

$H(\xi, \gamma, k, u)=\int_{y_{1}=0}^{\infty} \operatorname{Pr}\left(X_{2}>\xi \gamma X_{1}+k, X_{1}+X_{2}>u-y_{1}\right) f_{Y_{1}}\left(y_{1}\right) d y_{1}$

when $u<k$

$H^{-}(\xi, \gamma, k, u)=\int_{y_{1}=0}^{u} \operatorname{Pr}\left(X_{2}>\xi \gamma X_{1}+k\right) f_{Y_{1}}\left(y_{1}\right) d y_{1}+$

$\int_{y_{1}=u}^{\infty} \operatorname{Pr}\left(X_{2}>\xi \gamma X_{1}+k\right) f_{Y_{1}}\left(y_{1}\right) d y_{1}$

$H^{-}(\xi, \gamma, k, u)=\operatorname{Pr}\left(X_{2}>\xi \gamma X_{1}+k\right) \int_{y_{1}=0}^{\infty} f_{Y_{1}}\left(y_{1}\right) d y_{1}$

$H^{-}(\xi, \gamma, k, u)=F(\xi, \gamma, k) \times 1$

$H^{-}(\xi, \gamma, k, u)=\frac{\lambda_{h_{1}} e^{-k \lambda_{h_{2}}}}{\xi \gamma \lambda_{h_{2}}+\lambda_{h_{1}}}$

when $u>k$

$H^{+}(\xi, \gamma, k, u)=\int_{y_{1}=0}^{u-k} \underbrace{\operatorname{Pr}\left(X_{2}>\xi \gamma X_{1}+k, X_{1}+X_{2}>u-y_{1}\right)}_{G(\xi, \gamma, k, u)} \times$

$f_{Y_{1}}\left(y_{1}\right) d y_{1}+\int_{y_{1}=u-k}^{u} \operatorname{Pr}\left(X_{2}>\xi \gamma X_{1}+k\right) f_{Y_{1}}\left(y_{1}\right) d y_{1}+$

$\int_{y_{1}=u}^{\infty} \operatorname{Pr}\left(X_{2}>\xi \gamma X_{1}+k\right) f_{Y_{1}}\left(y_{1}\right) d y_{1}$

$=\int_{y_{1}=0}^{u-k} G(\xi, \gamma, k, u) f_{Y_{1}}\left(y_{1}\right) d y_{1}+\operatorname{Pr}\left(X_{2}>\xi \gamma X_{1}+k\right) \int_{y_{1}=u-k}^{\infty} f_{Y_{1}}\left(y_{1}\right) d y_{1}$

$=\int_{y_{1}=0}^{u-k} G(\xi, \gamma, k, u) f_{Y_{1}}\left(y_{1}\right) d y_{1}+\frac{\lambda_{h_{1}} e^{-k \lambda_{h_{2}}} e^{-(u-k) \lambda_{g_{1}}}}{\xi \gamma \lambda_{h_{2}}+\lambda_{h_{1}}}$

Let us first evaluate $G(\xi, \gamma, k, u)$

Let $u-y_{1}=v$ and conditioning $G(\xi, \gamma, k, u)$ on $X_{1}$
$G(\xi, \gamma, k, v)=\int_{x_{1}=0}^{\infty} \operatorname{Pr}\left(X_{2}>\xi \gamma x_{1}+k, X_{2}>v-x_{1}\right) f_{X_{1}}\left(x_{1}\right) d x_{1}$

$G(\xi, \gamma, k, v)=\int_{x_{1}=0}^{\infty} \operatorname{Pr}\left(X_{2}>\max \left(\xi \gamma x_{1}+k, v-x_{1}\right)\right) f_{X_{1}}\left(x_{1}\right) d x_{1}$

$\xi \gamma x_{1}+k=v-x_{1} \rightarrow x_{1}=\frac{v-k}{1+\xi \gamma}$

$G(\xi, \gamma, k, v)=\int_{x_{1}=0}^{\frac{v-k}{1+\xi \gamma}} F_{X_{2}}\left(v-x_{1}\right) f_{X_{1}}\left(x_{1}\right) d x_{1}+$

$\int_{x_{1}=\frac{v-k}{1+\xi \gamma}}^{\infty} F_{X_{2}}\left(\xi \gamma x_{1}+k\right) f_{X_{1}}\left(x_{1}\right) d x_{1}$

$G(\xi, \gamma, k, v)=\int_{x_{1}=0}^{\frac{v-k}{1+\xi \gamma}} \lambda_{h_{1}} e^{-v \lambda_{h_{2}}} e^{-\left(\lambda_{h_{1}}-\lambda_{h_{2}}\right) x_{1}} d x_{1}+$

$\int_{x_{1}=\frac{\nu-k}{1+\xi \gamma}}^{\infty} \lambda_{h_{1}} e^{-k \lambda_{h_{2}}} e^{-\left(\lambda_{h_{1}}+\xi \gamma \lambda_{h_{2}}\right) x_{1}} d x_{1}$

Performing the integration and after some algebraic calculation, we can find that,

$G(\xi, \gamma, k, v)=\frac{\lambda_{h_{1}} e^{-v \lambda_{h_{2}}}}{\left(\lambda_{h_{1}}-\lambda_{h_{2}}\right)}+e^{-\frac{\left(\lambda_{h_{1}}+\xi \gamma \lambda_{h_{2}}\right) v}{(1+\xi \gamma)}} e^{\frac{k\left(\lambda_{h_{1}}-\lambda_{h_{2}}\right)}{(1+\xi \gamma)}} \times$

$\left(\frac{\lambda_{h_{1}}}{\left(\lambda_{h_{1}}+\xi \gamma \lambda_{h_{2}}\right)}-\frac{\lambda_{h_{1}}}{\left(\lambda_{h_{1}}-\lambda_{h_{2}}\right)}\right)$

Now, putting the value of $v=u-y_{1}$ in $G(\xi, \gamma, k, v)$, we get,

$G(\xi, \gamma, k, u)=\frac{\lambda_{h_{1}} e^{-\lambda_{h_{2}} u}}{\left(\lambda_{h_{1}}-\lambda_{h_{2}}\right)} e^{\lambda_{h_{2}} y_{1}}+\left(\frac{\lambda_{h_{1}}}{\left(\lambda_{h_{1}}+\xi \gamma \lambda_{h_{2}}\right)}-\right.$

$\left.\frac{\lambda_{h_{1}}}{\left(\lambda_{h_{1}}-\lambda_{h_{2}}\right)}\right) e^{\frac{k\left(\lambda_{h_{1}}-\lambda_{h_{2}}\right)}{(1+\xi \gamma)}} e^{-\frac{\left(\lambda_{h_{1}}+\xi \gamma \lambda_{h_{2}}\right) u}{(1+\xi \gamma)}} e^{\frac{\left(\lambda_{h_{1}}+\xi \gamma \lambda_{h_{2}}\right) y_{1}}{(1+\xi \gamma)}}$

Now,

$H^{+}(\xi, \gamma, k, u)=\int_{y_{1}=0}^{u-k} G(\xi, \gamma, k, u) f_{Y_{1}}\left(y_{1}\right) d y_{1}+\underbrace{\frac{\lambda_{h_{1}} e^{-k \lambda_{h_{2}}} e^{-(u-k) \lambda_{g_{1}}}}{\xi \gamma \lambda_{h_{2}}+\lambda_{h_{1}}}}_{I_{1}}$

$H^{+}(\xi, \gamma, k, u)=\int_{y_{1}=0}^{u-k} G(\xi, \gamma, k, u) \lambda_{g_{1}} e^{-\lambda_{g_{1}} y_{1}} d y_{1}+I_{1}$

$H^{+}(\xi, \gamma, k, u)=\int_{y_{1}=0}^{u-k} \frac{\lambda_{h_{1}} e^{-\lambda_{h_{2}} u}}{\left(\lambda_{h_{1}}-\lambda_{h_{2}}\right)} e^{\lambda_{h_{2}} y_{1}} \lambda_{g_{1}} e^{-\lambda_{g_{1}} y_{1}} d y_{1}+$

$\int_{y_{1}=0}^{u-k}\left(\frac{\lambda_{h_{1}}}{\left(\lambda_{h_{1}}+\xi \gamma \lambda_{h_{2}}\right)}-\frac{\lambda_{h_{1}}}{\left(\lambda_{h_{1}}-\lambda_{h_{2}}\right)}\right) e^{\frac{k\left(\lambda_{h_{1}}-\lambda_{h_{2}}\right)}{(1+\xi \gamma)}} e^{-\frac{\left(\lambda_{h_{1}}+\xi \gamma \lambda_{h_{2}}\right) u}{(1+\xi \gamma)}} \times$

$e^{\frac{\left(\lambda_{h_{1}}+\xi \gamma \lambda_{h_{2}}\right) y_{1}}{(1+\xi \gamma)}} \lambda_{g_{1}} e^{-\lambda_{g_{1}} y_{1}} d y_{1}+I_{1}$

Again, performing the integration and after some algebraic calculation, we can find that,

$H^{+}(\xi, \gamma, k, u)=\frac{\lambda_{h_{1}} \lambda_{g_{1}} e^{-\lambda_{h_{2}} u}}{\left(\lambda_{h_{1}}-\lambda_{h_{2}}\right)\left(\lambda_{g_{1}}-\lambda_{h_{2}}\right)}-\frac{\lambda_{h_{1}} \lambda_{g_{1}} e^{\left(\lambda_{g_{1}}-\lambda_{h_{2}}\right) k} e^{-\lambda g_{1} u}}{\left(\lambda_{h_{1}}-\lambda_{h_{2}}\right)\left(\lambda_{g_{1}}-\lambda_{h_{2}}\right)}+$

$\left(\frac{\lambda_{h_{1}} \lambda_{g_{1}}}{\left(\lambda_{h_{1}}+\xi \gamma \lambda_{h_{2}}\right)}-\frac{\lambda_{h_{1}} \lambda_{g_{1}}}{\left(\lambda_{h_{1}}-\lambda_{h_{2}}\right)}\right) \frac{e^{\frac{k\left(\lambda_{h_{1}}-\lambda_{h_{2}}\right)}{(1+\xi \gamma)}} e^{-\frac{\left(\lambda_{h_{1}}+\xi \gamma \lambda_{h_{2}}\right) u}{(1+\xi \gamma)}}(1+\xi \gamma)}{\left(\lambda_{g_{1}}-\lambda_{h_{1}}\right)+\left(\lambda_{g_{1}}-\lambda_{h_{2}}\right) \xi \gamma}$

$-\left(\frac{\lambda_{h_{1}} \lambda_{g_{1}}}{\left(\lambda_{h_{1}}+\xi \gamma \lambda_{h_{2}}\right)}-\frac{\lambda_{h_{1}} \lambda_{g_{1}}}{\left(\lambda_{h_{1}}-\lambda_{h_{2}}\right)}\right) \frac{(1+\xi \gamma) e^{-\lambda_{g_{1}} u} e^{\left(\lambda_{g_{1}}-\lambda_{h_{2}}\right) k}}{\left(\lambda_{g_{1}}-\lambda_{h_{1}}\right)+\left(\lambda_{g_{1}}-\lambda_{h_{2}}\right) \xi \gamma}+$

$\underbrace{\frac{\lambda_{h_{1}} e^{k\left(\lambda_{g_{1}}-\lambda_{h_{2}}\right)} e^{-\lambda_{g_{1}} u}}{\xi \gamma \lambda_{h_{2}}+\lambda_{h_{1}}}}_{I_{1}}$ 
Now, putting,

$$
\begin{aligned}
& A_{S_{2}}=\frac{\lambda_{h_{1}} \lambda_{g_{1}}}{\left(\lambda_{h_{1}}-\lambda_{h_{2}}\right)\left(\lambda_{g_{1}}-\lambda_{h_{2}}\right)} \\
& B_{S_{2}}=\frac{\lambda_{h_{1}} e^{k\left(\lambda_{g_{1}}-\lambda_{h_{2}}\right)}}{\xi \gamma \lambda_{h_{2}}+\lambda_{h_{1}}}-\frac{\lambda_{h_{1}} \lambda_{g_{1}} e^{\left(\lambda_{g_{1}}-\lambda_{h_{2}}\right) k}}{\left(\lambda_{h_{1}}-\lambda_{h_{2}}\right)\left(\lambda_{g_{1}}-\lambda_{h_{2}}\right)}- \\
& \left(\frac{\lambda_{h_{1}} \lambda_{g_{1}}}{\left(\lambda_{h_{1}}+\xi \gamma \lambda_{h_{2}}\right)}-\frac{\lambda_{h_{1}} \lambda_{g_{1}}}{\left(\lambda_{h_{1}}-\lambda_{h_{2}}\right)}\right) \frac{(1+\xi \gamma) e^{\left(\lambda_{g_{1}}-\lambda_{h_{2}}\right) k}}{\left(\lambda_{g_{1}}-\lambda_{h_{1}}\right)+\left(\lambda_{g_{1}}-\lambda_{h_{2}}\right) \xi \gamma} \\
& C_{S_{2}}=\left(\frac{\lambda_{h_{1}} \lambda_{g_{1}}}{\left(\lambda_{h_{1}}+\xi \gamma \lambda_{h_{2}}\right)}-\frac{\lambda_{h_{1}} \lambda_{g_{1}}}{\left(\lambda_{h_{1}}-\lambda_{h_{2}}\right)}\right) \frac{e^{\frac{k\left(\lambda_{h_{1}}-\lambda_{h_{2}}\right)}{(1+\xi \gamma)}}(1+\xi \gamma)}{\left(\lambda_{g_{1}}-\lambda_{h_{1}}\right)+\left(\lambda_{g_{1}}-\lambda_{h_{2}}\right) \xi \gamma}
\end{aligned}
$$

Therefore,

$H^{+}(\xi, \gamma, k, u)=A_{S_{2}} e^{-\lambda_{h_{2}} u}+B_{S_{2}} e^{-\lambda_{g_{1}} u}+C_{S_{2}} e^{-\frac{\left(\lambda_{h_{1}}+\xi \gamma \lambda_{h_{2}}\right) u}{(1+\xi \gamma)}}$

Now,

$Q(\xi, \gamma, k, t)=\int_{y_{2}=0}^{\infty} \operatorname{Pr}\left(X_{2}>\xi \gamma X_{1}+k,\left(X_{1}+X_{2}+Y_{1}\right)>\frac{t}{y_{2}}-y_{2}\right) f_{Y_{2}}\left(y_{2}\right) d y_{2}$

Now,

$$
\begin{aligned}
& \frac{t}{y_{2}}-y_{2}=k \rightarrow y_{2}=y_{2}^{*}=\frac{-k+\sqrt{k^{2}+4 t}}{2} \\
& =\int_{y_{2}=0}^{y_{*}^{*}} H^{+}(\xi, \gamma, k, u) f_{Y_{2}}\left(y_{2}\right) d y_{2}+\int_{y_{2}=y_{2}^{*}}^{\sqrt{t}} \operatorname{Pr}\left(X_{2}>\xi \gamma X_{1}+k\right) f_{Y_{2}}\left(y_{2}\right) d y_{2}+ \\
& \int_{y_{2}=\sqrt{t}}^{\infty} \operatorname{Pr}\left(X_{2}>\xi \gamma X_{1}+k\right) f_{Y_{2}}\left(y_{2}\right) d y_{2} \\
& =\int_{y_{2}=0}^{y_{2}^{*}} H^{+}(\xi, \gamma, k, u) f_{Y_{2}}\left(y_{2}\right) d y_{2}+\operatorname{Pr}\left(X_{2}>\xi \gamma X_{1}+k\right) \int_{y_{2}=y_{2}^{*}}^{\infty} f_{Y_{2}}\left(y_{2}\right) d y_{2} \\
& Q(\xi, \gamma, k, t)=\int_{y_{2}=0}^{y_{2}^{*}}\left(A_{S_{2}} e^{-\lambda_{h_{2}} u}+B_{S_{2}} e^{-\lambda_{g_{1}} u}+C_{S_{2}} e^{-\frac{\left(\lambda_{h_{1}}+\xi \gamma \lambda_{2}\right) u}{(1+\xi \gamma)}}\right) \\
& \times \lambda_{g_{2}} e^{-\lambda_{g_{2}} y_{2}} d y_{2}+\frac{\lambda_{h_{1}} e^{-k \lambda_{h_{2}}}}{\xi \gamma \lambda_{h_{2}}+\lambda_{h_{1}}} e^{-\lambda_{g_{2}} y_{2}^{*}}
\end{aligned}
$$

Putting the value of $u=\frac{t}{y_{2}}-y_{2}$

$$
\begin{aligned}
& Q(\xi, \gamma, k, t)=\int_{y_{2}=0}^{y_{2}^{*}}\left(\left(A_{S_{2}} \lambda_{g_{2}} e^{-\frac{\lambda_{h_{2}} t}{y_{2}}-\left(\lambda_{g_{2}}+\lambda_{h_{2}}\right) y_{2}}\right)+\right. \\
& \left(B_{S_{2}} \lambda_{g_{2}} e^{-\frac{\lambda g_{1} t}{y_{2}}-\left(\lambda_{g_{2}}-\lambda_{g_{1}}\right) y_{2}}\right)+ \\
& \left.\left(C_{S_{2}} \lambda_{g_{2}} e^{-\frac{\left(\lambda_{h_{1}}+\xi \gamma \lambda_{h_{2}}\right) t}{(1+\xi \gamma) y_{2}}-\left(\frac{\left(\lambda_{g_{2}}-\lambda h_{1}\right)+\left(\lambda g_{2}-\lambda h_{2}\right) \xi \gamma}{(1+\xi \gamma)}\right) y_{2}}\right)\right) d y_{2}+\frac{\lambda_{h_{1}} e^{-k \lambda h_{2}}}{\xi \gamma \lambda h_{2}+\lambda_{h_{1}}} e^{-\lambda g_{2} y_{2}^{*}}
\end{aligned}
$$$$
Q(\xi, \gamma, k, t)=A_{S_{2}} \lambda_{g_{2}} \sum_{n=0}^{\infty} \frac{(-1)^{n}}{n !}\left(\lambda_{g_{2}}+\lambda_{h_{2}}\right)^{n}\left(y_{2}^{*}\right)^{n+1} E_{n+2}\left(\frac{\lambda_{h_{2}} t}{y_{2}^{*}}\right)+
$$$$
B_{S_{2}} \lambda_{g_{2}} \sum_{n=0}^{\infty} \frac{(-1)^{n}}{n !}\left(\lambda_{g_{2}}-\lambda_{g_{1}}\right)^{n}\left(y_{2}^{*}\right)^{n+1} E_{n+2}\left(\frac{\lambda_{g_{1}} t}{y_{2}^{*}}\right)+
$$$$
C_{S_{2}} \lambda_{g_{2}} \sum_{n=0}^{\infty} \frac{(-1)^{n}}{n !}\left(\frac{\left(\lambda_{g_{2}}-\lambda_{h_{1}}\right)+\left(\lambda_{g_{2}}-\lambda_{h_{2}}\right) \xi \gamma}{(1+\xi \gamma)}\right)^{n}\left(y_{2}^{*}\right)^{n+1}
$$$$
E_{n+2}\left(\frac{\left(\lambda_{h_{1}}+\xi \gamma \lambda_{h_{2}}\right) t}{(1+\xi \gamma) y_{2}^{*}}\right)+\frac{\lambda_{h_{1}} e^{-k \lambda_{h_{2}}}}{\xi \gamma \lambda_{h_{2}}+\lambda_{h_{1}}} e^{-\lambda_{g_{2}} y_{2}^{*}}
$$$$
\text { Therefore, } P_{S_{2}}^{\text {Out }}=1-Q(\xi, \gamma, k, t)
$$

Substituting $Q(\xi, \gamma, k, t)$ above, we get the final expression as in Equation 19.

This completes the proof of Theorem 2 .

\section{REFERENCES}

[1] S. Mumtaz, A. Alsohaily, Z. Pang, A. Rayes, K. F. Tsang, and J. Rodriguez, "Massive internet of things for industrial applications: Addressing wireless iiot connectivity challenges and ecosystem fragmentation," IEEE Industrial Electronics Magazine, vol. 11, no. 1, pp. 28-33, 2017.

[2] J. Howell, "Number of connected iot devices will surge to 125 billion by 2030," IHS markit says, 2017.

[3] Z. Wu, K. Lu, C. Jiang, and X. Shao, "Comprehensive study and comparison on $5 \mathrm{~g}$ noma schemes," IEEE Access, vol. 6, pp. 18511$18519,2018$.

[4] J. G. Andrews, S. Buzzi, W. Choi, S. V. Hanly, A. Lozano, A. C. Soong, and J. C. Zhang, "What will 5g be?" IEEE Journal on selected areas in communications, vol. 32, no. 6, pp. 1065-1082, 2014.

[5] S. Hu, B. Yu, C. Qian, Y. Xiao, Q. Xiong, C. Sun, and Y. Gao, "Nonorthogonal interleave-grid multiple access scheme for industrial internet of things in 5g network," IEEE Transactions on Industrial Informatics, vol. 14, no. 12, pp. 5436-5446, 2018.

[6] S. R. Islam, N. Avazov, O. A. Dobre, and K.-S. Kwak, "Power-domain non-orthogonal multiple access (noma) in $5 \mathrm{~g}$ systems: Potentials and challenges," IEEE Communications Surveys \& Tutorials, vol. 19, no. 2, pp. 721-742, 2016.

[7] Q. Wu, W. Chen, D. W. K. Ng, and R. Schober, "Spectral and energy-efficient wireless powered iot networks: Noma or tdma?" IEEE Transactions on Vehicular Technology, vol. 67, no. 7, pp. 6663-6667, 2018.

[8] S. R. Islam, M. Zeng, O. A. Dobre, and K.-S. Kwak, "Resource allocation for downlink noma systems: Key techniques and open issues," IEEE Wireless Communications, vol. 25, no. 2, pp. 40-47, 2018.

[9] Z. Ding, Z. Yang, P. Fan, and H. V. Poor, "On the performance of non-orthogonal multiple access in $5 \mathrm{~g}$ systems with randomly deployed users," IEEE signal processing letters, vol. 21, no. 12, pp. 1501-1505, 2014.

[10] Y. Huang, M. Liu, and Y. Liu, "Energy-efficient swipt in iot distributed antenna systems," IEEE Internet of Things Journal, vol. 5, no. 4, pp. 2646-2656, 2018.

[11] W. Guo, S. Zhou, Y. Chen, S. Wang, X. Chu, and Z. Niu, "Simultaneous information and energy flow for iot relay systems with crowd harvesting," IEEE Communications Magazine, vol. 54, no. 11, pp. 143-149, 2016.

[12] X. Lu, P. Wang, D. Niyato, D. I. Kim, and Z. Han, "Wireless networks with rf energy harvesting: A contemporary survey," IEEE Communications Surveys \& Tutorials, vol. 17, no. 2, pp. 757-789, 2014.

[13] X. Zhou, R. Zhang, and C. K. Ho, "Wireless information and power transfer: Architecture design and rate-energy tradeoff," IEEE Transactions on communications, vol. 61, no. 11, pp. 4754-4767, 2013.

[14] A. A. Nasir, X. Zhou, S. Durrani, and R. A. Kennedy, "Relaying protocols for wireless energy harvesting and information processing," IEEE Transactions on Wireless Communications, vol. 12, no. 7, pp. 3622-3636, 2013.

[15] A. Gupta, K. Singh, and M. Sellathurai, "Time-switching eh-based joint relay selection and resource allocation algorithms for multi-user multicarrier af relay networks," IEEE Transactions on Green Communications and Networking, vol. 3, no. 2, pp. 505-522, 2019.

[16] A. Rauniyar, P. Engelstad, and O. N. Østerb, "Rf energy harvesting and information transmission based on power splitting and noma for iot relay systems," in 2018 IEEE 17th International Symposium on Network Computing and Applications (NCA). IEEE, 2018, pp. 1-8.

[17] T. Nguyen, P. Tran Tin, D. Ha, M. Voznak, P. Tran, M. Tran, and T.-L. Nguyen, "Hybrid tsr-psr alternate energy harvesting relay network over rician fading channels: Outage probability and ser analysis," Sensors, vol. 18, no. 11, p. 3839, 2018.

[18] Z. Yang, Z. Ding, Y. Wu, and P. Fan, "Novel relay selection strategies for cooperative noma," IEEE Transactions on Vehicular Technology, vol. 66, no. 11 , pp. $10114-10123,2017$.

[19] R. Jiao, L. Dai, J. Zhang, R. MacKenzie, and M. Hao, "On the performance of noma-based cooperative relaying systems over rician fading channels," IEEE Transactions on Vehicular Technology, vol. 66, no. 12, pp. 11 409-11413, 2017.

[20] Z. Ding, H. Dai, and H. V. Poor, "Relay selection for cooperative noma," IEEE Wireless Communications Letters, vol. 5, no. 4, pp. 416-419, 2016.

[21] N. T. Do, D. B. Da Costa, T. Q. Duong, and B. An, "A bnbf user selection scheme for noma-based cooperative relaying systems with swipt," IEEE Communications Letters, vol. 21, no. 3, pp. 664-667, 2016.

[22] A. Rauniyar, P. E. Engelstad, and O. N. Østerb $\varnothing$, "Performance analysis of rf energy harvesting and information transmission based on noma 
with interfering signal for iot relay systems," IEEE Sensors Journal, vol. 19, no. 17, pp. 7668-7682, 2019.

[23] M. F. Kader, M. B. Uddin, S. R. Islam, and S. Y. Shin, "Capacity and outage analysis of a dual-hop decode-and-forward relay-aided noma scheme," Digital Signal Processing, vol. 88, pp. 138-148, 2019.

[24] H. Tabassum, M. S. Ali, E. Hossain, M. Hossain, D. I. Kim et al., "Nonorthogonal multiple access (noma) in cellular uplink and downlink: Challenges and enabling techniques," arXiv preprint arXiv:1608.05783, 2016.

[25] H. Tabassum, M. S. Ali, E. Hossain, M. J. Hossain, and D. I. Kim, “Uplink vs. downlink noma in cellular networks: Challenges and research directions," in 2017 IEEE 85th Vehicular Technology Conference (VTC Spring). IEEE, 2017, pp. 1-7.

[26] X. Yue, Y. Liu, S. Kang, A. Nallanathan, and Z. Ding, "Exploiting full/half-duplex user relaying in noma systems," IEEE Transactions on Communications, vol. 66, no. 2, pp. 560-575, 2017.

[27] M. Xu, F. Ji, M. Wen, and W. Duan, "Novel receiver design for the cooperative relaying system with non-orthogonal multiple access," IEEE Communications Letters, vol. 20, no. 8, pp. 1679-1682, 2016.

[28] Z. Yang, Z. Ding, P. Fan, and N. Al-Dhahir, "The impact of power allocation on cooperative non-orthogonal multiple access networks with swipt," IEEE Transactions on Wireless Communications, vol. 16, no. 7, pp. 4332-4343, 2017.

[29] J. Tang, J. Luo, M. Liu, D. K. So, E. Alsusa, G. Chen, K.-K. Wong, and J. A. Chambers, "Energy efficiency optimization for noma with swipt," IEEE Journal of Selected Topics in Signal Processing, vol. 13, no. 3 , pp. 452-466, 2019.

[30] S. K. Zaidi, S. F. Hasan, and X. Gui, "Swipt-aided uplink in hybrid nonorthogonal multiple access," in 2018 IEEE Wireless Communications and Networking Conference (WCNC). IEEE, 2018, pp. 1-6.

[31] B. Rankov and A. Wittneben, "Spectral efficient protocols for halfduplex fading relay channels," IEEE Journal on selected Areas in Communications, vol. 25, no. 2, pp. 379-389, 2007.

[32] M. Choi, D.-J. Han, and J. Moon, "Bi-directional cooperative noma without full csit," IEEE Transactions on Wireless Communications, vol. 17 , no. 11 , pp. $7515-7527,2018$.

[33] X. Yue, Y. Liu, S. Kang, A. Nallanathan, and Y. Chen, "Modeling and analysis of two-way relay non-orthogonal multiple access systems," IEEE Transactions on Communications, vol. 66, no. 9, pp. 3784-3796, 2018.

[34] M. F. Kader, M. B. Uddin, M. A. L. Sarker, and S. Y. Shin, "Bidirectional relaying using non-orthogonal multiple access," Physical Communication, 2019.

[35] 3rd Generation Partnership Project (3GPP), "Study on downlink multiuser superposition transmission for LTE, TSG RAN Meeting 67," Tech. Rep. RP-150496, Mar 2015.

[36] D. K. Nguyen, D. N. K. Jayakody, S. Chatzinotas, J. S. Thompson, and $\mathrm{J}$. Li, "Wireless energy harvesting assisted two-way cognitive relay networks: Protocol design and performance analysis," Ieee Access, vol. 5, pp. 21 447-21460, 2017.

[37] K. Mimis, D. R. Gibbins, S. Dumanli, and G. T. Watkins, "The ant and the elephant: ambient rf harvesting from the uplink," IET Microwaves, Antennas \& Propagation, vol. 11, no. 3, pp. 386-393, 2016.

[38] B. Medepally and N. B. Mehta, "Voluntary energy harvesting relays and selection in cooperative wireless networks," IEEE Transactions on Wireless Communications, vol. 9, no. 11, pp. 3543-3553, 2010.
[39] Y. Gu and S. Aissa, "Rf-based energy harvesting in decode-and-forward relaying systems: Ergodic and outage capacities," IEEE Transactions on Wireless Communications, vol. 14, no. 11, pp. 6425-6434, 2015.

[40] A. Rauniyar, P. Engelstad, and O. Østerbø, "Rf energy harvesting and information transmission based on noma for wireless powered iot relay systems," Sensors, vol. 18, no. 10, p. 3254, 2018.

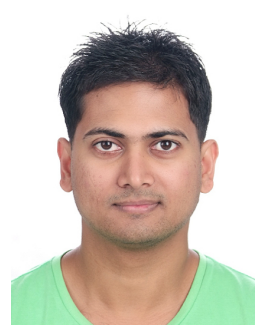

ASHISH RAUNIYAR is currently working as a PhD Research Fellow with the Autonomous Systems and Sensor Technologies Research Group, Department of Technology Systems, Faculty of Mathematics and Natural Sciences, University of Oslo and Autonomous Systems and Networks (ASN) Research Group, Department of Computer Science, Oslo Metropolitan University, Norway. He was a graduate research assistant at Wireless Emerging Networking System (WENS) Lab, where he completed his Master degree in IT Convergence Engineering at Kumoh National Institute of Technology, South-Korea. He is a recipient of Best Paper Award at IEEE 28th ITNAC Conference, 2018, Sydney, Australia. His main research area includes Internet of Things, Machine Learning, Wireless Communications and Computer Networking.

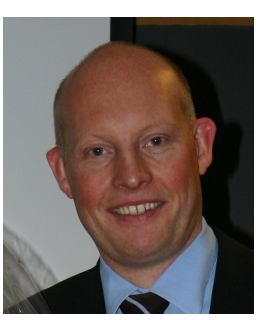

PAAL E. ENGELSTAD received the Bachelor Degree in Physics from the Norwegian University of Science and Technology (NTNU) in 1993, the Master Degree (Hons.) in Physics from NTNU/Kyoto University, Japan, in 1994, the Ph.D. Degree in Computer Science from the University of Oslo in 2005. He is currently a Full Professor with Oslo Metropolitan University. He is also a Research Scientist at Norwegian Defence Research Establishment and a Professor with the Autonomous Systems and Sensor Technologies Research Group, Department of Technology Systems, University of Oslo. He holds a number of patents and has been publishing a number of papers over the past years. His current research interests include fixed, wireless and adhoc networking, cybersecurity, machine learning, and distributed and autonomous systems.

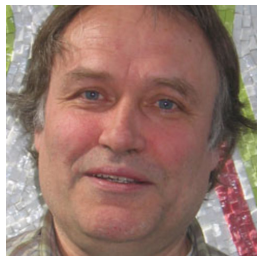

OLAV N. ØSTERB Ø received his M.Sc. in Applied Mathematics from the University of Bergen in 1980 and his Ph.D. from NTNU in 2004. He joined Telenor in 1980 and has more than 30 years of experience in telecom research. Activities in recent years have been related to QoS and performance analysis. Current research topics include traffic modeling, analysis of interference in radio networks, scheduling, traffic differentiation, and M2M. 